\title{
Continuous Hawking-Page transitions in Einstein-scalar gravity
}

\author{
Umut Gürsoy \\ Institute for Theoretical Physics, Utrecht University, \\ Leuvenlaan 4, 3584 CE Utrecht, The Netherlands \\ E-mail: u.gursoy@uu.nl
}

ABSTRACT: We investigate continuous Hawking-Page transitions in Einstein's gravity coupled to a scalar field with an arbitrary potential in the weak gravity limit. We show that this is only possible in a singular limit where the black-hole horizon marginally traps a curvature singularity. Depending on the subleading terms in the potential, a rich variety of continuous phase transitions arise. Our examples include second and higher order, including the Berezinskii-Kosterlitz-Thouless type. In the case when the scalar is dilaton, the condition for continuous phase transitions lead to (asymptotically) linear-dilaton background. We obtain the scaling laws of thermodynamic functions, as well as the viscosity coefficients near the transition. In the limit of weak gravitational interactions, the bulk viscosity asymptotes to a universal constant, independent of the details of the scalar potential. As a byproduct of our analysis we obtain a one-parameter family of kink solutions in arbitrary dimension $d$ that interpolate between AdS near the boundary and linear-dilaton background in the deep interior. The continuous Hawking-Page transitions found here serve as holographic models for normal-to superfluid transitions.

KEYwords: Classical Theories of Gravity, Black Holes, Gauge-gravity correspondence, Black Holes in String Theory

ARXiv EPRINT: 1007.0500 


\section{Contents}

1 Introduction 1

2 Weak phase transitions and small black-holes 5

2.1 Generalities 5

2.2 Conditions for presence of continuous transitions 8

$\begin{array}{ll}2.3 & \text { Linear dilaton background in NCST } \\ \end{array}$

$\begin{array}{ll}2.4 \text { The problem of singularity } & 12\end{array}$

3 Classification of continuous transitions $\quad 12$

$\begin{array}{lll}3.1 & \text { General results } & 12\end{array}$

$\begin{array}{ll}3.2 & \text { Derivation in a coordinate invariant approach } \\ \end{array}$

4 Scaling of thermodynamic functions $\quad \mathbf{1 5}$

$\begin{array}{lll}4.1 & \text { Free energy } & 15\end{array}$

$\begin{array}{lll}4.2 & \text { Fine-tuning the potential } & 17\end{array}$

$\begin{array}{lll}4.3 & \text { Entropy and specific heat } & 17\end{array}$

$\begin{array}{ll}4.4 \text { Speed of sound } & 18\end{array}$

4.5 An order of limits issue and divergence in the dilaton 18

5 Viscosities and dispersion $\quad 19$

$\begin{array}{ll}5.1 \text { Shear deformations } & 19\end{array}$

$\begin{array}{ll}5.2 \text { Bulk deformations } & 20\end{array}$

$\begin{array}{ll}5.3 \text { Universality at } T_{c} & 21\end{array}$

5.4 Scaling of viscosities near $T_{c} \quad 22$

5.5 Dispersion relation of pressure waves $\quad 22$

$6 \quad$ UV geometry and analytic kink solutions $\quad 23$

6.1 Two-exponent potentials 23

$\begin{array}{lll}6.2 & \text { Three-exponent potentials } & 25\end{array}$

7 Stringy corrections $\quad 26$

$\begin{array}{lll}7.1 & \text { Higher derivative corrections } & 27\end{array}$

$\begin{array}{lll}7.21 / N^{2} \text { corrections } & 29\end{array}$

$\begin{array}{llr}8 & \text { Discussion } & 29\end{array}$

A The scalar variables and reduction of the Einstein-scalar system 32

B Derivation of thermodynamics from the scalar variables 33

C Sub-leading corrections to the geometry near $T_{c} \quad 33$ 


\section{Introduction}

Thermodynamics of radiation in asymptotically Anti-de-Sitter spaces is quite interesting. It was shown by Hawking and Page in [1] that, as one heats up a gas of gravitons in the global AdS space-time, one finds a phase transition into nucleation of black-holes at a finite temperature $T_{c} \sim \ell^{-1}$ where $\ell$ is the AdS radius. At low temperatures, the only equilibrium solution to Einstein's equations with asymptotically AdS boundary condition is thermal radiation. Above a certain temperature $T_{\min } \sim \ell^{-1}$ a "small black-hole" and a "big black-hole" saddle coexist with the thermal gas (TG). Right above $T_{\min }$ these solutions have free energy larger than the radiation, therefore they eventually tunnel into thermal radiation. However, above a certain value $T_{c}>T_{\min }$ the big black-hole solution becomes the global minimum and the thermal gas instead becomes quantum mechanically unstable against tunneling into the big black-hole. ${ }^{1}$ This is the so-called Hawking-Page transition in asymptotically AdS space-times.

Witten in [2] proposed that the Hawking-Page transition in the $A d S_{5} \times S^{5}$ solution of IIB string theory corresponds to a confinement-deconfinement type transition in $\mathcal{N}=4$ Yang-Mills theory on $S^{3}$ with radius $R \sim \ell$, through the AdS/CFT corespondence [3-5]. There is a transition at some $T_{c} \sim R^{-1} \sim \ell^{-1}$ and it only occurs in the large color limit $N \rightarrow \infty$, where the number of degrees of freedom becomes infinite. A more interesting case is when the field theory is defined on $R^{3}$, which can be obtained as the large radius limit of the sphere $R \rightarrow \infty$. Clearly $T_{c} \rightarrow 0^{+}$in this case. The corresponding statement in the dual field theory is that the $\mathcal{N}=4 \mathrm{sYM}$ theory is conformal, and when placed on $S^{1} \times S^{3}$ with $S^{1}$ being the time-circle with radius $1 / T$, the thermodynamic quantities can only depend on the dimensionless ratio $T R$. Therefore, there cannot be any interesting thermodynamics in the flat space limit.

The situation changes, when there is an additional intrinsic scale in the problem that does happen in non-conformal theories. A basic example is pure Yang-Mills theory with gauge group $\mathrm{SU}(N)$. The theory has running coupling, hence a dynamical scale $\Lambda$ is generated through dimensional transmutation. Indeed, lattice studies provide strong evidence for a second order deconfinement transition for $N=2$ and a first order deconfinement transition for $N>2$ in 3 space dimensions roughly at $T_{c} \sim \Lambda$.

\footnotetext{
${ }^{1}$ The small BH solution has negative specific heat and it is always subdominant with respect to the big $\mathrm{BH}$ and TG saddles. It will play no role in what follows.
} 
By a natural generalization of the ideas in [2], one expects that Hawking-Page type transitions in asymptotically AdS space-times dual to Yang-Mills type non-conformal theories $^{2}$ should correspond to confinement-deconfinement transitions in these theories. In [6], it was shown that the simple set-up of Einstein gravity coupled to a scalar field $\Phi$ with some potential $V(\Phi)$ allows for asymptotically AdS type solutions with a non-trivial intrinsic scale $\Lambda$ that is generated due to the running scalar field. It was proposed in $[7,8]$ that the ground state (zero T) solution generates color confinement in the dual field theory, if and only if the IR asymptotics of the potential obeys,

$$
V(\Phi) \rightarrow e^{\frac{4}{3} \Phi} \Phi^{P}, \quad P \geq 0, \quad \text { as } \Phi \rightarrow \infty,
$$

in 5 dimensions. A background that solves a potential of the form (1.1) in the IR and becomes asymptotically AdS in the UV indeed exhibits a Hawking-Page transition that corresponds to the deconfinement transition in the dual gauge theory.

In fact, the thermodynamics in the generic case $P>0$ is very similar to the global AdS case that we described above: There is always a thermal gas solution saddle at every $\mathrm{T}$. This is the only equilibrium configuration up to a certain $T_{\min }$. Above $T_{\min }$ there are two black-hole solutions in addition to the thermal gas: the small and the big BHs, just as in global AdS. At a certain $T_{c}>T_{\min }$ the big $\mathrm{BH}$ solution becomes the global minimum of the theory. This temperature corresponds to the deconfinement temperature in the proposed dual YM theory, where the glueballs deconfine their glue contents. For $P>0$, one can quite generally show [9] that, there is a finite latent heat and the transition is of first order.

In this paper, we ask the general question, "under what conditions gravity exhibits continuous Hawking-Page type phase transitions?" By the word continuous, we mean second or higher order transitions where the entropy is continuous across the transition. In other words, the latent heat vanishes. Regardless of the matter content of the gravity theory, the basic condition for a continuous HP transition can easily be obtained in the classical limit $G_{D} \rightarrow 0$. In this limit we arrive at the (more or less obvious) fact that, $a$ continuous transition in this limit can only occur in a singular region of space-time, where the BH horizon marginally traps a curvature singularity. This is easily understood: in the weak curvature limit, the contribution of thermal radiation to the entropy is negligible, thus the entropy of the TG solution is very small (zero in the strict $G_{D} \rightarrow 0$ limit). Then, continuity of entropy requires that the horizon size of the $\mathrm{BH}$ solution becomes very small as one approaches the transition temperature from above.

To be more definite, we approach the problem of continuous transitions in the context of Einstein's gravity coupled to a scalar. In fact, it was already observed in [6] that, the aforementioned HP transition may be continuous in the marginal limit $P=0$ in (1.1). Here, we focus in this particular case in more detail and also generalize the analysis to arbitrary $d+1$ dimensions. Our general result can be stated as follows: there exists a continuous Hawking-Page transition in $d+1$ dimensional Einstein-scalar theory if and only if

$$
V(\Phi) \rightarrow e^{\frac{4}{d-1} \Phi}\left(1+V_{\text {sub }}(\Phi)\right), \quad \text { as } \Phi \rightarrow \infty
$$

\footnotetext{
${ }^{2}$ By a Yang-Mills type theory we mean a gauge-theory that exhibits quark confinement in the ground state.
} 
where $V_{\text {sub }}$ denotes subleading corrections that vanish in the deep IR. We also find that these corrections determine the type of the transition: For an exponential fall-off in $V_{\text {sub }}$, one finds nth order transitions for $n \geq 2$. In this case the free energy difference scale as,

$$
\Delta F \sim\left(T-T_{c}\right)^{n}, \quad \text { as } T \rightarrow T_{c}^{+} .
$$

On the other hand, for a power-law fall-off, a Berezinskii-Kosterlitz-Thouless type scaling arises:

$$
\Delta F \sim e^{-A t^{-\frac{1}{\alpha}}}, \quad \text { as } T \rightarrow T_{c}^{+},
$$

where $\alpha>1$ is a constant.

A good indication that the marginal case $P=0$, corresponds to continuous transitions is obtained by investigating the equilibrium solutions of $V_{\text {conf } f}$. As mentioned above, for $P>$ 0 generically there are three solutions at high temperatures: the thermal gas, the big and the small black-holes. In the marginal limit $P \rightarrow 0$ the small black-hole solution disappears. Therefore, in the high $\mathrm{T}$ regime there exist only the TG and the big BH branches. In fact, in this case $T_{\min }$ and $T_{c}$ can be shown to coincide [9] and the thermodynamics becomes much simpler than the generic $P>0$ case: The low $\mathrm{T}$ (high $\mathrm{T}$ ) phase is dominated by the TG (big $\mathrm{BH}$ ) saddle and these phases are separated by a Hawking-Page transition at $T_{c}$. This is precisely what one expects in a continuous phase transition: At $T_{c}$ the new saddle is created and immediately becomes the global minimum of the free energy. Indeed the small BH solution can be considered as a kink that connects the TG and the big BH solutions which can only exist in first order transitions. In the limit where the transition becomes continuous this kink should disappear.

As we mentioned above, in the classical limit, the continuous transitions generically occur in a singular region where the horizon area becomes very small. In the particular example of the Einstein-scalar gravity with potential (1.1) and with $P=0$, this region corresponds to $\Phi_{h} \rightarrow \infty$ where $\Phi_{h}$ is the value of the scalar on the horizon. ${ }^{3}$ In this limit, it was shown in [9] that the $\mathrm{BH}$ and the TG solutions become degenerate, that is indeed another basic property of second or higher order transitions. In fact, in the strict classical limit $G_{D} \rightarrow 0$, the transition point corresponds to $\Phi_{c}=\infty$.

Clearly we cannot trust Einstein's gravity in this singular region. On the other hand we would like to study the scaling of thermodynamic functions with $T-T_{c}$, an information contained in this singular region. We avoid this problem here by fine-tuning the sub-leading term in (1.2) such that the region $T \lesssim T_{c}$ corresponds to a wide range $\Phi_{h} \in\left(\Phi_{s}, \infty\right)$ where $\Phi_{s}$ is the value of $\Phi$ where the scaling behavior in (1.3) or (1.4) sets in. This is clearly a crude method that we can resort to, only in a phenomenological approach where we use the freedom in the choice of the potential to hide the problem of singularity. This method serves our purposes in this paper, that is to work out the qualitative behavior of thermodynamic functions in a continuous HP transition. However, a more detailed future study should take into account the $\alpha^{\prime}$ corrections by investigating continuous HP transitions in the full string theory.

\footnotetext{
${ }^{3}$ It can also be viewed as the horizon location in a coordinate system where the radial direction is given by $\Phi$.
} 
Studying the IR geometry of the solutions to (1.2) we arrive at another interesting result: the $\mathrm{BH}$ and the $\mathrm{TG}$ geometries both asymptote to a linear-dilaton geometry, in the case when the scalar field is the dilaton. More precisely, the ground state (TG solution) that uniquely follows from the potential (1.2) is asymptotically linear-dilaton in the deep interior of the geometry. At the same time, the BH geometry approaches to the TG geometry in the limit $T \rightarrow T_{c}$. Therefore both of the saddles are of linear-dilaton type in this region of space. It is well-known that the linear-dilaton geometry is an $\alpha^{\prime}$ exact solution to the full string theory. This observation may prove important in studying the analogous problem in the full string theory.

In the limit where we can ignore gravitational interactions and higher derivative corrections, Einstein's gravity predicts certain universal results around a continuous transition. One such quantity is the value of the bulk viscosity per entropy as $T \rightarrow T_{c}$. We find that this value is constant across the transition: ${ }^{4}$

$$
\frac{\zeta}{s} \rightarrow \frac{1}{2 \pi(d-1)}, \quad \text { as } T \rightarrow T_{c} .
$$

The result is universal in the sense that it is independent of the details of the theory such as the choice for the scalar potential $V(\Phi)$, the transition temperature $T_{c}$ etc., and it is only subject to $\alpha^{\prime}$ corrections in a gravity theory with higher derivative terms.

We should mention that although this work can be regarded as an investigation purely in gravity, we have holographic applications in mind. In this paper, we shall not specify the expected dual field theory. In fact most of our findings will not depend on the UV geometry either. Yet, we would like to have an asymptotically AdS geometry in the UV, to be able use the prescriptions of the AdS/CFT duality in holographic applications. A particularly interesting such application is studied in a companion paper [11], where we propose that the continuous phase transitions found here are holographically related to normal-to-superfluid transitions in spin-models.

In the course of searching for backgrounds with AdS asymptotics, we also obtain a one-parameter family of analytic kink solutions that flow from an AdS extremum to the linear-dilaton background in the string frame.

In the next section, we list the general conditions for the Einstein-scalar theory to exhibit a continuous Hawking-Page transition. In section 3, we present the classification of the transitions that follow from different fall-off behavior of $V_{s u b}$ in (1.2). In this section, we also present a coordinate invariant method to study thermodynamics in Einstein-scalar gravity, that was first constructed in [9]. Here we generalize this method here to arbitrary dimensions. Section 4 is devoted to derivation of the scaling behavior of thermodynamic functions: the free energy, the entropy, the specific heat and the speed of sound. In section 5 we study the shear and bulk deformations around the black-hole solution and study the viscosity-entropy ratios $\eta / s$ and $\zeta / s$ in the transition region. In section 6 we construct the aforementioned analytic kink soutions. Finally in section 7 , we briefly mention what type of corrections are expected in our findings when the set-up is embedded in full string

\footnotetext{
${ }^{4}$ See $[10]$ for another example where $\zeta / s$ is found to be constant.
} 
theory. Finally we present a discussion on our results and an outlook for future directions in the last section. Six appendices detail our calculations.

\section{Weak phase transitions and small black-holes}

\section{$2.1 \quad$ Generalities}

In this section we determine the requirements for presence of continuous phase transitions in the Einstein-scalar theory in $d+1$ dimensions, defined by the action:

$$
\mathcal{A}=\frac{1}{16 \pi G_{D}} \int d^{d+1} x \sqrt{-g}\left(R-\xi(\partial \Phi)^{2}+V(\Phi)\right)+\text { G.H. }
$$

We keep the normalization of the scalar kinetic term $\xi>0$ as a free parameter for later convenience. The last term, that we shall not need to specify here, is the Gibbons-Hawking term on the boundary. $G_{D}$ is the Newton's constant. The total number of bulk dimensions is denoted by $D=d+1$.

There are only two types of backgrounds at finite $\mathrm{T}$ (Euclidean time is compactified), with Poincaré symmetries in $d-1$ spatial dimensions, and and additional U(1) symmetry in the Euclidean time direction: the thermal graviton gas,

$$
d s^{2}=e^{2 A_{0}(r)}\left(d r^{2}+d x_{d-1}^{2}+d t^{2}\right), \quad \Phi=\Phi_{0}(r),
$$

and the black-hole,

$$
d s^{2}=e^{2 A(r)}\left(f^{-1}(r) d r^{2}+d x_{d-1}^{2}+d t^{2} f(r)\right), \quad \Phi=\Phi(r) .
$$

We define the coordinate system such that the boundary is located at $r=0$. For the potentials $V$ that we consider in this paper, there is a curvature singularity in the deep interior, at $r=r_{s}$. In (2.2), $\mathrm{r}$ runs up to singularity $r_{s}$. In (2.3) there is a horizon that cloaks this singularity at $r_{h}<r_{s}$ where $f\left(r_{h}\right)=0$. $\mathrm{t}$ is the Euclidean time that is identified as $t \sim t+1 / T$. This defines the temperature $\mathrm{T}$ of the associated thermodynamics. In the black-hole solution, the relation between the temperature and $r_{h}$ is obtained in the standard way, by assuming that the near horizon geometry is conical and the conical singularity is absent:

$$
4 \pi T=-f^{\prime}\left(r_{h}\right)
$$

This identifies $\mathrm{T}$ and the surface gravity in the $\mathrm{BH}$ solution.

The geometries (2.2) and (2.3) are the only backgrounds with the boundary condition that $A(r) \rightarrow A_{0}(r), \Phi(r) \rightarrow \Phi_{0}(r)$ and $f(r) \rightarrow 1$ near the boundary. ${ }^{5}$ We are interested in determining the necessary and sufficient conditions for existence of a continuous HawkingPage type transition between (2.2) and (2.3). We define a continuous phase transition as the one where the entropy difference vanishes $\Delta S\left(T_{c}\right)=0$ at the transition. This defines

\footnotetext{
${ }^{5}$ This is somewhat rough, see [9] for precise matching conditions in the variable $r$, near the boundary. The easiest way to implement the matching is to consider a coordinate frame where $\Phi$ is the radial variable and identifies $\Phi=\Phi_{0}$ in the entire range of the two solutions. Then the only nontrivial condition is $A(\Phi) \rightarrow A_{0}(\Phi)$ and $f(\Phi) \rightarrow 1$ near the boundary. This is what we will do in section 3 .
} 
phase transitions of second and higher order, including infinite order, such as the BKT transition $[12,13] .^{6}$

Thermodynamics is determined by the Gibbs free energy that is given by the on-shell value of the action (2.1). Taking the difference between the on-hell values on the BH and the TG solutions removes possible divergences due to infinite volume of the geometries and yields the regularized action:

$$
T^{-1} \Delta G(T)=\mathcal{A}(B H)-\mathcal{A}(T G)+\cdots
$$

where the ellipsis refer to corrections that are $\mathcal{O}\left(G_{D}\right)$ suppressed with respect to the first term.

The Newton's constant in (2.1) is important in determining the overall size of the thermodynamic functions. It also plays an important role in the correspondence between gravity and a dual field theory, where $G_{D}$ should be proportional to the number of degrees of freedom in the dual quantum field theory. This is most clear when the proposed dual theory is is a gauge theory with $N$ colors:

$$
\frac{1}{16 \pi G_{D}}=M_{p}^{d-1} N^{2}
$$

where $M_{p}$ is a "normalized" Planck scale, that is generally of the same order as the typical curvature of the background. ${ }^{7}$ On the gravity side the factor $\mathrm{N}$ arises in a similar fashion to the $A d S_{5} \times S^{5}$ case. Here it is supposed to be proportional to the RR $d+1$ form flux on the geometry [7]. As explained in [7] the space-filling flux can be integrated out by replacing it with its equation of motion in the action, and it only contributes to the potential $V(\Phi)$. The potential $V$ that is written in (2.1) is supposed to include this contribution. This procedure is completely analogous to the 5-dimensional case discussed in [7] and we refer the reader to this paper for details.

Clearly, in order to be able to ignore interactions between bulk fields one needs to take the large $\mathrm{N}$ limit. This can easily be done when the scalar is dilaton: in an asymptotically AdS solution where the boundary value of the dilaton is constant $\Phi_{0}$, the large $\mathrm{N}$ limit is given by sending the dilaton $\Phi_{0} \rightarrow-\infty$, and the flux $N \rightarrow \infty$ such that $e^{\Phi_{0}} N=$ const.

In this work, we will mostly be interested in the scaling of the various thermodynamic quantities with $\mathrm{T}$ and not in the overall size of these quantities. For that purpose, it is more convenient to factor out the overall $G_{D}$ dependence by dividing the free energy by $V_{d-1} N^{2}$ where $V_{d-1}$ is the volume of the spatial part, and $N^{2}$ is defined by (2.6). We, thus define the normalized free energy density,

$$
\Delta F(T)=\frac{1}{V_{d-1} T^{-1} N^{2}}(\mathcal{A}(B H)-\mathcal{A}(T G))+\cdots \equiv F_{0}(T)+\delta F(T) .
$$

\footnotetext{
${ }^{6}$ See [14] and [15] for recent papers where the BKT scaling was observed in the context of condensed matter applications of holography. These papers consider quantum phase transitions however, where the scaling is not in $\mathrm{T}$ but in a quantum coupling.

${ }^{7}$ For example, in the improved holographic QCD models [6], it is given by $M_{p}=\left(45 \pi^{2}\right)^{-\frac{1}{3}} \ell^{-1}$ where $\ell$ is the curvature radius of the asymptotically AdS geometry near the boundary.
} 
Here $F_{0}$ is defined as the leading $\mathcal{O}\left(N^{0}\right)$ piece in the free energy density, and $\delta F$ refers to the $\mathcal{O}\left(N^{-2}\right)$ corrections. The large limit $N \rightarrow \infty\left(G_{D} \rightarrow 0\right)$ defines a saddle point approximation from the gravitational point of view. The corrections that we denote by $\delta F$ in (2.7) arise, for finite but small $G_{D}$ from the determinant of gravitational fluctuations around the saddle:

$$
\delta F(T)=\operatorname{Tr} \log O_{B H}-\operatorname{Tr} \log O_{T G},
$$

where $O$ is the d'Alembertian operator of the fluctuations around the saddle geometries. ${ }^{8}$

Although, we shall mostly be interested in the leading $F_{0}$ term in (2.7), we note the following important consequence of the $\delta F$ correction: Using the 1st law, the entropy (density) difference is given by, ${ }^{9}$

$$
\Delta S=S_{B H}-S_{T G}=-\frac{d F_{0}}{d T}-\frac{d \delta F}{d T},
$$

where we normalize the entropy the same way as in (2.7). The $S_{T G}$ term above has no leading $\mathcal{O}\left(N^{0}\right)$ piece because the TG geometry has no horizon. Therefore, one has,

$$
\Delta S=S_{B H}-\frac{d \delta F}{d T}
$$

We shall ignore the $1 / N^{2}$ corrections throughout the paper and drop the second term above, hence the entropy (density) difference of the dual theory is entirely determined by the black-hole entropy that is therefore given by the horizon size at $r_{h}$ :

$$
\Delta S=S_{B H}=\frac{e^{(d-1) A\left(r_{h}\right)}}{4 G_{D} N^{2}}=4 \pi M_{p}^{d-1} e^{(d-1) A\left(r_{h}\right)},
$$

where we used the relation (2.6) in the second equation.

Therefore, we learn from $(2.11)$ that - up to $1 / N^{2}$ corrections that we neglect in this paper - a necessary condition for a continuous phase transition is that the black-hole becomes infinitesimal at $T_{c}$. This is an infinitesimal black-hole ${ }^{10}$ with vanishing classical horizon size. ${ }^{11}$ In the backgrounds we consider there is a single curvature singularity at $r=r_{c}$. Precisely at $T_{c}$, the singularity is marginally trapped by the black-hole, and $T_{c}$ corresponds to the temperature where the horizon approaches the singularity $r_{h} \rightarrow r_{c} \cdot{ }^{12}$

There are further conditions for the existence of a phase transition as explained in the following. Before we present the derivation in the next subsection, let us state the

\footnotetext{
${ }^{8}$ In principle, for finite $G_{D}$, one has to make sure that this difference is free of divergences. By the boundary condition that BH asymptotes to TG near the boundary, the two spectra in the UV are guaranteed to be the same (this can be checked by a WKB analysis), hence the difference should be convergent.

${ }^{9}$ The backgrounds that we consider in this paper satisfy the required energy conditions and the laws of thermodynamics should apply.

${ }^{10}$ Not to be confused with the aforementioned "small black-hole" saddle which is a black-hole with a negative specific heat. In all of the examples we consider in this paper, we only consider black-holes with non-negative specific heat.

${ }^{11}$ Note that the black-hole at $T_{c}$ should be non-extremal, but with vanishing enthalpy.

${ }^{12}$ One may consider the possibility of string corrections at this point. We will discuss such possible corrections in the last section.
} 
final result: In the Einstein-scalar gravity, there exists a continuous phase transition into nucleation of black-holes if and only if the scalar potential $V(\Phi)$ has the asymptotic behavior,

$$
V(\Phi) \rightarrow e^{2 \sqrt{\frac{\xi}{d-1}} \Phi}, \quad \Phi \rightarrow \infty .
$$

This result is of course subject to field redefinitions in the action (2.1). Below, we present a derivation where we stick to the form of the action given in (2.1).

\subsection{Conditions for presence of continuous transitions}

We are interested in a Hawking-Page type phase transition when the thermal gas of gravitons (2.2) becomes unstable against nucleation of black holes (2.3) and we ask the question under what conditions this transition is higher than first order? The thermodynamic requirements are clear:

i. $\exists T_{c}$ such that $0<T_{c}<\infty$ and $\Delta F\left(T_{c}\right)=0$,

ii. $\Delta S\left(T_{c}\right)=0$.

Solution of these conditions for a generic scalar potential $V$ in (2.1) may seem rather arbitrary. This is not the case however. As we show below, the conditions above imply the specific form (2.12) for the asymptotic form of $V$.

Before solving the conditions above, first we have to define the various thermodynamic functions in terms of geometric quantities. We solve the equations of motion and derive the thermodynamics in a coordinate independent approach in section 3.2. Here we shall first work with the coordinate frame - that we call the r-frame - defined by the metrics $(2.2)$ and (2.3) for simplicity. In the r-frame, one derives the following Einstein and scalar equations of motion from (2.1):

$$
\begin{array}{r}
A^{\prime \prime}-A^{2}+\frac{\xi}{d-1} \Phi^{\prime 2}=0, \\
f^{\prime \prime}+(d-1) A^{\prime} f^{\prime}=0, \\
(d-1) A^{\prime 2} f+A^{\prime} f^{\prime}+A^{\prime \prime} f-\frac{V}{d-1} e^{2 A}=0 .
\end{array}
$$

One easily solves (2.14) to obtain the "blackness function" $f(r)$ in terms of the scale factor as,

$$
f(r)=1-\frac{\int_{0}^{r} e^{-(d-1) A}}{\int_{0}^{r_{h}} e^{-(d-1) A}} .
$$

Then the temperature of the $\mathrm{BH}$ is given by equation (2.4):

$$
T^{-1}=4 \pi e^{(d-1) A\left(r_{h}\right)} \int_{0}^{r_{h}} e^{-(d-1) A(r)} d r .
$$

The difference between the entropy densities of the $\mathrm{BH}$ and the TG solutions is given by the $\mathrm{BH}$ entropy density (2.11) up to $1 / N^{2}$ corrections that we ignore from now on:

$$
\Delta S=\frac{1}{4 G_{D} N^{2}} e^{(d-1) A\left(r_{h}\right)}
$$


The difference in the free energy densities (2.7) can equally well be evaluated by integrating the first law of thermodynamics $(2.9),[9]:{ }^{13}$

$$
\Delta F\left(r_{h}\right)=-\frac{1}{4 G_{D} N^{2}} \int_{r_{c}}^{r_{h}} e^{(d-1) A\left(\tilde{r}_{h}\right)} \frac{d T}{d \tilde{r}_{h}} d \tilde{r}_{h}
$$

Here $r_{c}$ is the value of the horizon size that corresponds to the phase transition, $T\left(r_{c}\right)=T_{c}$, where the difference in free energies should vanish. We note that, dependence on $r_{h}$ in the integrand of (2.19) is two-fold: There is the explicit dependence on the variable $r_{h}$ and there is the implicit dependence that arise because the function $A(r)$ itself changes as $r_{h}$ is varied. ${ }^{14}$ As we showed in [9], this implicit dependence on $r_{h}$ is suppressed in the limit $r_{h}$ close to the singularity $r_{s}$. This is a fact that will prove important in the analysis of the next section.

It was demonstrated in [8] that, for any domain-wall type geometry of the form (2.2) with the condition that the scale factor obeys $A^{\prime}\left(r_{0}\right)<0$ near the boundary, ${ }^{15}$ the possible asymtotics in the deep interior can be classified as:

A. An asymptotically AdS geometry in the deep interior as $r \rightarrow \infty$,

B. A curvature singularity at finite $r=r_{s}$ where $\phi_{0} \rightarrow \infty$ and $A_{0} \rightarrow-\infty$, and

C. A curvature singularity at $r=\infty$ where $\phi_{0} \rightarrow \infty$ and $A_{0} \rightarrow-\infty$.

We want to determine which type of geometry above admits a Hawking-Page transition by satisfying the conditions $i$ and ii stated in the beginning of this section.

We first focus on the condition ii: For a continuous phase transition, one should require that the entropy difference (2.18) should vanish at the transition point $r_{c}$. Then, from the classification above we learn that $r_{c}$ should correspond to either of the singular points $r=r_{s}$ in case ii, or $r=\infty$ in the cases i or iii, because that is the only point where the scale factor vanishes, so does $S$ by $(2.18) .{ }^{16}$

Now we consider the condition ii above. In order to maintain a finite $T_{c}$, from (2.17) one should require that the metric function near the singularity should behave linearly:

$$
A(r) \rightarrow-A_{\infty} r+\cdots, \quad r \rightarrow \infty
$$

where $A_{\infty}$ is a constant and the ellipsis may involve (powers of) log's or terms that are sub-dominant as $r \rightarrow \infty$. This singles out the deep-interior geometry of the type $\mathrm{C}$ above. Then, the rest of the condition $\mathrm{i}$ is satisfied automatically: The fact that $\Delta F$ vanishes at $r_{c}=\infty$ follows immediately from the definition $F\left(T_{c}\right)=E\left(T_{c}\right)-T_{c} S\left(T_{c}\right)$. The enthalpy

\footnotetext{
${ }^{13}$ We ignore the $\delta F$ term in (2.9) that is subleading in $N$.

${ }^{14}$ This is clear when solving (2.13) by specifying the boundary conditions at $r_{h}$. See section 3.2 for more detail.

${ }^{15}$ This is the only technical requirement in this section. We note that asymptotically AdS geometries are of this type.

${ }^{16}$ We refer to [9] for a derivation of the fact that the $\mathrm{BH}(\mathrm{TG})$ metric functions $A\left(A_{0}\right)$ become asymptotically the same at the singular points. Thus requirement that $\exp A_{0}$ vanishes is equivalent to the requirement that $\exp A$ vanishes.
} 
piece vanish because the entropy vanishes and $T_{c}$ is finite. The energy also vanish by the fact that in the limit $T \rightarrow T_{c}$ which corresponds to $r_{c}=\infty$ the BH becomes of zero size, hence the ADM mass vanishes. ${ }^{17}$

This is of course in accord with the first law of thermodynamics (2.19) where we tacitly made the assumption that $\Delta F$ vanishes at $r_{c}$. Here, in addition to this we learned that, for a continuous transition, one needs $r_{c}=\infty$ and one should require (2.20) in the deep-interior. This latter condition can be expressed in a coordinate-invariant way, as a condition on the asymptotic form of the scalar potential $V(\Phi)$, as follows: The Einstein equation (2.13) determines the asymptotic form of the scalar field:

$$
\Phi(r) \rightarrow \sqrt{\frac{d-1}{\xi}} A_{\infty} r+\cdots, \quad r \rightarrow \infty
$$

where, as in (2.20) the ellipsis may involve logs. Now, the asymptotic form of the potential $V$ is determined by the scalar e.o.m., (2.15), using (2.20), and inverting (2.21) in favor of $\Phi$ as,

$$
V(\Phi) \rightarrow V_{\infty} e^{2 \sqrt{\frac{\xi}{d-1}} \Phi}\left(1+V_{\text {sub }}(\Phi)\right), \quad \Phi \rightarrow \infty
$$

where $V_{\infty}$ is a constant.

The term $V_{\text {sub }}$ in (2.22) is a sub-leading piece: $V_{\text {sub }} \rightarrow 0$ as $\Phi \rightarrow \infty$. This piece will be important in determining the order of the phase transition in the next section. It is interesting to note that, even if log terms were present in (2.20) and (2.21), they conspire to produce exactly the single exponential (with no $\log \Phi$ corrections in the exponential) in the leading asymptotics.

The only dimensionful parameter in the problem is $V_{\infty}$ in (2.22). Indeed, $V$ has dimensions of $\ell^{-2}$ where $\ell$ is a typical radius of curvature of the TG geometry, for example , it is the AdS radius when the TG geometry is asymptotically AdS. Thus, all of the other dimensionful quantities above, i.e. $A_{\infty}, \Phi_{\infty}$ and $T_{c}$ should be determined in terms of $V_{\infty}$ :

$$
T_{c} \propto A_{\infty} \propto \Phi_{\infty} \propto \ell^{-1} \propto \sqrt{V_{\infty}}
$$

We shall determine the proportionality constants precisely in the next section.

To summarize: in the Einstein-scalar set-up, continuous phase transitions of order $\geq 2$ arise with and only with the asymptotics (2.22). The transition happens at $r_{h}=r_{c}=\infty$ where the entropy, the energy and the free energy vanishes, as the black hole has vanishing classical horizon in this limit. ${ }^{18}$ However, the surface gravity, hence the temperature remains finite. Finally, the value of the transition temperature is controlled by constant $V_{\infty}$ in $(2.23)$.

\footnotetext{
${ }^{17}$ See [9] for a careful computation of the ADM mass.

${ }^{18}$ As explained in section 2.1 above, vanishing of the BH entropy and free energy density is equivalent to the vanishing of the entropy and free energy difference between the BH and the TG backgrounds, to leading order in $1 / N^{2}$.
} 
A comment: We have a $\mathrm{BH}$ geometry that has vanishing mass at $T_{c}$, to leading order in the $1 / N^{2}$ expansion. Yet the temperature $T_{c}$ is finite. One should ask how a massless object can have finite temperature? Does it radiate? In fact our situation is very similar to what happens in the massless limit of linear dilaton black-holes [16]. As the authors of [16] show in the special case of a massless linear dilaton $\mathrm{BH}$, although the temperature remains finite, the radiation from the event horizon vanishes in the massless limit, hence there is no contradiction. In our case as well, one can show that the radiation vanishes in the massless limit $r_{h} \rightarrow \infty$. We will show this in section 5 by computing the shear and the bulk viscosity coefficients $\eta$ and $\zeta$ and showing that they vanish in the limit $r_{h} \rightarrow \infty$. However, we also show that the non-trivial and interesting quantities are not the bare viscosity coefficients $\eta$ and $\zeta$ but the normalized ones $\eta / S$ and $\zeta / S$ which remain finite in the limit $T \rightarrow T_{c}$. We refer the reader to section 5 for more details.

\subsection{Linear dilaton background in NCST}

Now, we consider the special case when the scalar $\Phi$ is the dilaton of $(d+1)$ non-critical string theory. The NS-NS action of NCST in $d+1$ dimensions read,

$$
S \propto \int d^{d+1} x \sqrt{-g_{s}} e^{-2 \Phi}\left(R_{s}+4(\partial \Phi)^{2}+\frac{\delta c}{\ell_{s}^{2}}+\cdots\right),
$$

where we only show the relevant terms in the two-derivative sector, the ellipsis refer to higher order terms in the derivative expansion. $\delta c$ refers to the deficit central charge in $d+1$ dimensions, e.g. it is $9-d$ in a parent $10 \mathrm{D}$ critical string theory. The NS-NS two-form $\mathrm{B}$ is chosen to constant, hence it does not contribute to the equations of motion. The subscript s refers to string frame quantities.

One passes to the Einstein frame by the Weyl transformation $g_{s}^{\mu \nu}=e^{4 \Phi /(d-1)} g^{\mu \nu}$. The transformation produces an Einstein-frame action of the form (2.1) with the normalization $\xi$ fixed as,

$$
\xi_{s}=\frac{4}{d-1} .
$$

In this case the requirement for weak phase transitions (2.22) become,

$$
V \rightarrow V_{\infty} e^{\frac{4}{d-1} \Phi}+\cdots
$$

Interestingly, the form of the dilaton potential (in the Einstein frame), (2.22) that follows from the condition for existence of continuous phase transitions, precisely coincides with the dilaton potential that one obtains in the NCST after passing to the Einstein frame. At the same time, we know that there is an $\alpha^{\prime}$-exact solution to the NCST, that is the linear dilaton background. [17]. Indeed the asymptotic form of the solutions we found in the previous section, both for the TG (2.2) and the BH (2.3) backgrounds, ${ }^{19}$ become linear dilaton geometries in the limit $r_{h} \rightarrow \infty$, if one passes to the string frame:

$$
d s_{s}^{2}=\left(d r^{2}+d x_{d}^{2}\right)(1+\cdots), \quad \Phi(r)=\frac{d-1}{2} A_{\infty} r(1+\cdots)
$$

\footnotetext{
${ }^{19}$ In the BH case the blackness function $f(r)$ approaches 1 as the horizon size becomes smaller [9].
} 
where the corrections denoted by the ellipsis vanish as $r \rightarrow \infty$. The precise form of these corrections depend on $V_{\text {sub }}$ in (2.22).

The crucial fact is that when $\Phi$ is the dilaton of NCST, the string-frame scale factor $A_{s}=A+2 \Phi /(d-1)$ vanishes when $V$ has the form (2.26), thus one obtains a linear-dilaton geometry in the deep-interior, in the string frame. Because the linear-dilaton is an exact solution to non-critical string theory in all orders in $\alpha^{\prime}$ we expect that the leading term in the condition (2.22) for presence of continuous transitions is $\alpha^{\prime}$-exact. We have more to say on this in section 7 .

In passing, we note that in this particular case of the D-dim. NCST (with $\xi=4 / d-1$ ), the coefficients $A_{\infty}, \Phi_{\infty}$ in (2.20) and (2.21), and the transition temperature $T_{c}$ can be expressed in terms of the parameter $V_{\infty}$ in a precise way (see appendix $\mathrm{D}$ for derivation):

$$
A_{\infty}=\frac{2}{d-1} \Phi_{\infty}=\frac{4 \pi}{d-1} T_{c}=\frac{\sqrt{V_{\infty}}}{d-1} .
$$

This last equation, in particular, grants us with direct control over the transition temperature by adjusting the amplitude of the scalar potential in the asymptotic region. Clearly, this is very much desired in phenomenological applications.

\subsection{The problem of singularity}

We showed above that existence of a continuous Hawking-Page transition in the Einsteinscalar theory requires a singular geometry with a curvature singularity in the deep interior. The transition happens at the point when the horizon marginally traps the singularity.

Of course, the Einstein's theory breaks down as one approaches this singular limit and one can no longer control the calculations. In fact, in the string frame we do not have to worry about the curvature corrections precisely around the transition region because the curvature invariants can be shown to vanish in this limit, see appendix E. Instead the issue is that the dilaton becomes very large hence the effective string coupling becomes strong in this region and our assumption of ignoring the gravitational interactions cannot be justified. We can avoid this problem, if we have the means of fine-tuning the asymptotic value of $e^{\Phi}$ near the boundary to be parametrically small. This is what we shall do in the following: we will adjust the dilaton potential $V$ in such a way that the approach to the singularity is slow enough. More precisely, we will choose the subleading terms in the potential such that the vicinity around $T_{c}$ corresponds is away from the large $\Phi$ region. How to manage this, is explained in section 4.2. In section 6 we construct explicit examples where we can show that this fine-tuning is equivalent to choosing the asymptotic value of the dilaton to be small.

\section{Classification of continuous transitions}

\subsection{General results}

In this section we investigate the role of the sub-leading asymptotics in the scalar potential $V_{\text {sub }}(\Phi)$ in determining the type of the phase transition. We shall not be completely general, but only focus on two interesting cases where $V_{\text {sub }}$ is either an exponential or a power-law. 
We state the results before detailing their derivation. We consider two different classes of asymptotics:

$$
\begin{aligned}
& \text { Case i : } \quad V_{\text {sub }}=C e^{-\kappa \Phi}, \quad \kappa>0, \quad \Phi \rightarrow \infty \\
& \text { Case ii : } \quad V_{\text {sub }}=C \Phi^{-\alpha}, \quad \alpha>0, \quad \Phi \rightarrow \infty
\end{aligned}
$$

Furthermore, we define the normalized temperature,

$$
t=\frac{T-T_{c}}{T_{c}}
$$

The asymptotics (3.1) and (3.2) the free energy density difference,

$$
\begin{array}{cl}
\text { Order } n \text { transition : } \Delta F(t) \sim t^{n}, & \text { for } \kappa=\frac{\sqrt{\xi(d-1)}}{n-1}, n \geq 2 \\
\text { Infinite order transition : } \Delta F(t) \sim e^{-A t^{-\frac{1}{\alpha}}}, & \text { for } \alpha>0 .
\end{array}
$$

In particular when $\alpha=2$ one obtains a BKT type scaling in the case (3.2):

$$
\Delta F(t) \sim e^{-A t^{-\frac{1}{2}}}, \quad \alpha=2 .
$$

Another special case is when the scalar field is the dilaton in NCST where the normalization of the kinetic term in $(2.1)$ becomes $\xi=4 /(d-1)$. Then, in the case (3.1) above, one has an nth order transition for $\kappa=2 /(n-1)$.

\subsection{Derivation in a coordinate invariant approach}

We would like to derive the results (3.4) and (3.5) in an approach that is independent of the chosen coordinate system, where one only needs to specify the asymptotics of the scalar potential as in (3.1) and (3.2). In this section we introduce such an approach. The derivation of the thermodynamic properties based on this approach is presented in the next section.

Such a coordinate independent approach was introduced in terms of the so-called "scalar variables", in section 7 of $[9] .{ }^{20}$ The idea is to introduce variables that transform as scalars under the diffeomorphism that involve $r$ i.e. $r \rightarrow \tilde{r}(r)$. Then, one reduces the complicated Einstein-scalar system to a system of coupled first order equations for these scalar variables.

One can always show that all physical results, including the thermodynamic functions etc. will only depend on this reduced system.

In case of a neutral black-hole in $d+1$ dimensions, one only needs two such variables, that we call $X$ and $Y$ :

$$
X(\Phi) \equiv \frac{\gamma}{d} \frac{\Phi^{\prime}}{A^{\prime}}, \quad Y(\Phi) \equiv \frac{1}{d} \frac{f^{\prime}}{f A^{\prime}} .
$$

where the constant $\gamma$ is given by,

$$
\gamma=\sqrt{\frac{d \xi}{d-1}}
$$

\footnotetext{
${ }^{20}$ See also the "thermal superpotential" method in [9] that is equivalent to the scalar variables.
} 
These functions satisfy the following system of coupled first order equations:

$$
\begin{aligned}
& \frac{d X}{d \Phi}=-\gamma\left(1-X^{2}+Y\right)\left(1+\frac{1}{2 \gamma} \frac{1}{X} \frac{d \log V}{d \Phi}\right), \\
& \frac{d Y}{d \Phi}=-\gamma\left(1-X^{2}+Y\right) \frac{Y}{X} .
\end{aligned}
$$

We leave the derivation of these and other equations below to appendix A. These equations are solved by imposing boundary conditions at the horizon: Let us denote the value of the scalar at the horizon as $\Phi_{h}$. Then regularity of the horizon fixes the values of $X\left(\Phi_{h}\right)$ and $Y\left(\Phi_{h}\right)$ completely in terms of the scalar potential:

$$
\begin{aligned}
Y(\Phi) & =\frac{Y_{h}}{\Phi_{h}-\Phi}+Y_{1}+\mathcal{O}\left(\Phi_{h}-\Phi\right) \\
X(\Phi) & =X_{h}+X_{1}\left(\Phi_{h}-f\right)+\mathcal{O}\left(\Phi_{h}-\Phi\right)^{2}
\end{aligned}
$$

with

$$
X_{h}=-\frac{1}{2 \gamma} \frac{V^{\prime}\left(\Phi_{h}\right)}{V\left(\Phi_{h}\right)}, \quad Y_{h}=-\frac{X_{h}}{\gamma}, \quad X_{1}=-\frac{1}{4 \gamma}\left(\frac{V^{\prime \prime}\left(\Phi_{h}\right)}{V\left(\Phi_{h}\right)}-\frac{V^{\prime}\left(\Phi_{h}\right)^{2}}{V\left(\Phi_{h}\right)^{2}}\right), \quad Y_{1}=1-X_{h}^{2} .
$$

All other sub-leading terms are determined similarly by perturbative expansion near $\Phi_{h}$.

Having determined the functions $X$ and $Y$ by solving these eqs. the metric functions are obtained by the definitions (3.7) as:

$$
\begin{aligned}
& A(\Phi)=A_{0}+\frac{\gamma}{d} \int_{\Phi_{0}}^{\Phi} \frac{1}{X} d \tilde{\Phi} \\
& f(\Phi)=\exp \left[\gamma \int_{\Phi_{0}}^{\Phi} \frac{Y}{X} d \tilde{\Phi}\right]
\end{aligned}
$$

where $\Phi_{0}$ is the boundary value of the scalar field, and in the second equation we used the requirement that the metric function $f$ is unity at the boundary.

This reduction corresponds to setting $\Phi$ as the radial variable, with the hidden assumption that $r(\Phi)$ is a single valued function, i.e. $\Phi(r)$ is monotonically increasing.

Finally, one can invert (3.9) to express the scalar potential in terms of the scalar variables as,

$$
V(\Phi)=\frac{d(d-1)}{\ell^{2}}\left(1+Y-X^{2}\right) e^{-2 \gamma \int_{-\infty}^{\Phi}\left(X(t)-\frac{Y(t)}{2 X(t)}\right) d t} .
$$

Derivation of thermodynamic relations in terms of the scalar variables is presented in appendix B. Given the scalar potential $V(\Phi)$ one solves for $X(\Phi)$ in (3.9) and (3.10) obtains the thermodynamics of the corresponding $\mathrm{BH}$ solution from the following two equations.

$$
\begin{aligned}
\Delta F\left(\Phi_{h}\right) & =\frac{1}{4 G_{D}} \int_{\Phi_{h}}^{\infty} d \tilde{\Phi}_{h} e^{(d-1) A\left(\Phi_{h}\right)} \frac{d T}{d \tilde{\Phi}_{h}}, \\
T\left(\Phi_{h}\right) & =\frac{\ell}{4 \pi(d-1)} e^{A\left(\Phi_{h}\right)} V\left(\Phi_{h}\right) e^{\gamma \int_{\Phi_{0}}^{\Phi_{h}} X(\Phi) d \Phi} .
\end{aligned}
$$


The first one follows from the 1st law of thermodynamics (2.9) and the expression for the entropy (2.11). The second one follows from expressing (2.4) in terms of the scalar variables. We refer to appendix B for details.

The constant $\ell$ that appears in these equations is the length scale, (2.23), that is given by the curvature radius of the geometry at the boundary $\Phi_{0}$, which is in turn determined by the value of the potential at $\Phi_{0}$ :

$$
\ell^{2}=d(d-1) \frac{1-X\left(\Phi_{0}\right)^{2}}{V\left(\Phi_{0}\right)}
$$

For example, in case of pure AdS, the scalar should be constant, hence $X=0$ and from (3.19) follows the standard expression for the cosmological constant in $\mathrm{d}+1$ dimensions.

\section{Scaling of thermodynamic functions}

\subsection{Free energy}

Now, it is a matter of straightforward calculation to see how the free energy scales with temperature for asymptotics of the type (3.1) and (3.2) that hold in the region $1 \ll \Phi<\Phi_{h}$. One first solves $X$ and $Y$ from (3.9) and (3.10) and shows that,

$$
X(\Phi)=-\frac{1}{\sqrt{d}}+\delta X(\Phi), \quad Y(\Phi)=Y_{0}(\Phi)+\delta Y(\Phi)
$$

where $\delta X$ and $\delta Y$ are sub-leading with respect to the first terms in the limit $1 \ll \Phi<\Phi_{h}$.

It turns out that we do not need the precise expressions for $\delta X, Y_{0}$ and $\delta Y$ above to determine the type of the phase transition. However they become important, for example, in determining the viscosity coefficients around $T_{c}$. We present them, in appendix B.

One determines the scale factor $A(\Phi)$ from (3.14) as,

$$
A\left(\Phi_{h}\right)=-\frac{\gamma}{\sqrt{d}} \Phi_{h}-\gamma \int^{\Phi_{h}} \delta X(\Phi) d \Phi+\text { const. }
$$

The constant piece is unimportant in what follows and one can show that the second term is sub-leading with respect to the first one, see appendix $\mathrm{C}$

Substituting (4.1) and (4.2) in (3.18), one finds that all the dependence on $\delta X$ cancels out and the rate of approach of $\mathrm{T}$ toward $T_{c}$ is directly determined by $V_{\text {sub }}$. Using the definition of the normalized temperature (3.3), one finds:

$$
t=V_{\text {sub }}\left(\Phi_{h}\right)
$$

Now the free-energy as a function of $t$ is given by (3.17) and it can be rewritten as,

$$
\Delta F(t) \propto \int_{0}^{t} d \tilde{t} e^{(d-1) A(\tilde{t})}
$$


The dependence of the scale factor on $t$ should be found by inverting (4.3) and substituting in (the leading term of) (4.2): In the cases (3.1) and (3.2) one finds,

$$
\begin{array}{rlrl}
\text { Case i : } & A(t)=\frac{1}{\kappa} \sqrt{\frac{\xi}{d-1}} \log (t / C)+\cdots, & & t \rightarrow 0^{+} \\
\text {Case ii : } & A(t)=-\sqrt{\frac{\xi}{d-1}}(t / C)^{-\frac{1}{\alpha}+\cdots,} & t \rightarrow 0^{+},
\end{array}
$$

The free energy follows from (4.4) as

$$
\begin{aligned}
\text { Case i : } & \Delta F(t) \propto t^{\frac{\sqrt{\xi(d-1)}}{\kappa}+1}, & t \rightarrow 0^{+} \\
\text {Case ii : } & \Delta F(t) \propto e^{C^{\prime} t^{-\frac{1}{\alpha}}} t^{1+\frac{1}{\alpha}}, & t \rightarrow 0^{+},
\end{aligned}
$$

where $C^{\prime}=\sqrt{\xi(d-1)} C^{\frac{1}{\alpha}}$ in the second equation. We see that $\Delta F$ vanishes, as it should, for arbitrary but positive constants $\xi, \kappa$ and $\alpha$.

In the special case of

$$
\kappa=\sqrt{\xi(d-1)} /(n-1)
$$

in (4.7), one obtains an nth order transition in case i. Moreover, if the scalar field is dilaton, then $\xi$ is given by (2.25) and (4.9) reduces to,

$$
\kappa=\frac{2}{n-1}
$$

In this case the required asymptotics in (2.22) for an order-n transition becomes,

$$
V(\Phi) \rightarrow e^{\frac{4}{d-1} \Phi}+C e^{\frac{4 n-2 d-2}{(n-1)(d-1)}}, \quad \Phi \rightarrow \infty .
$$

For example, for a 3rd order phase transition in a dual $2+1$ dimensional theory, one should require,

$$
V(\Phi) \rightarrow e^{2 \Phi}+C e^{\Phi}, \quad \Phi \rightarrow \infty .
$$

On the other hand, the special case of $\alpha=2$ in (3.2) is a BKT type transition where the free energy scales like ${ }^{21}$

$$
F(t) \sim e^{\frac{-C^{\prime}}{\sqrt{t}}}
$$

We note that the setting is rich enough to cover different BKT type scalings that one encounters in the condensed-matter literature for different values of $\alpha$. For example, in the dislocation theory of melting in two-dimensional solids [18] one encounters a different value, $\alpha=2.70541 \ldots$ in (4.8).

We also note that this set-up covers other, more general type of divergent transitions where one of the derivatives of the free energy becomes not discontinuous, but diverges. For a recent reference, cf. [19]. Clearly, these type of transitions are ubiquitous in the case i above, for arbitrary real $\kappa$.

\footnotetext{
${ }^{21}$ One can also get rid of the power-like corrections in (4.8) by slightly modifying the asymptotics in (3.2) by adding log corrections, but we shall not consider this here.
} 


\subsection{Fine-tuning the potential}

Before continuing further, we would like to comment on the issue of the transition region coinciding with the singular region in Einstein's gravity, section 2.4. In this paper we are only interested in how the thermodynamic quantities scale with the reduced temperature $t$ and not in the overall size of these quantities. In this case, one way to avoid the problem of running into the singular region is to fine-tune the parameters of the potential $V(\Phi)$ to parametrically separate the singular region $\Phi_{h} \gg 1$ from the vicinity of temperatures where the scaling sets in, i.e. $t \ll 1$.

Equation (4.3) suggests an obvious way to manage this separation just by choosing the phenomenological parameter $C$ in (3.1) and (3.2), small enough. In the Einstein frame how small it should be chosen is determined by the demand that the size of the curvature $R \ell^{2}$ is not too large. ${ }^{22}$ Suppose we decide to trust Einstein's gravity up to the point where the curvature becomes some order 1 number $A$. Then, in the region where $V$ is approximately given by (2.22), one has,

$$
R \propto V_{\infty} e^{2 \sqrt{\frac{\xi}{d-1}} \Phi_{h}} \sim A / \ell^{2},
$$

where the proportionality constants are $\mathcal{O}(1)$. We refer the reader to appendix E for a derivation of the curvature invariants. Substituting this in (3.1) or (3.2) determines how small the constant $C$ should be chosen. In case 1, for example, one needs,

$$
C \ll\left(\frac{A}{V_{\infty} \ell^{2}}\right)^{\frac{\kappa \sqrt{d-1}}{2 \sqrt{\xi}}} .
$$

In our case $C$ is a phenomenological parameter that can be chosen arbitrarily small.

Another means to accomplish the separation of the transition region $t \approx 0$ and the singular region $\Phi_{h} \gg 1$ is to tune the boundary value of the dilaton to be very small. This can be done in an asymptotically AdS solution, see section 6 .

Either of these ways seem to be a crude way to avoid the problem and there is a prize that one pays. For example when we consider quantities given by ratios such as $\eta / S$ and $\zeta / S$, we will see that there is an ambiguity in determining their value at $T_{c}$.

\subsection{Entropy and specific heat}

As we focus on continuous transitions in this paper, the classical entropy difference vanishes exactly at the transition. ${ }^{23}$ The scaling of the entropy near $T_{c}$ is determined by $(2.11)$ and (4.5), (4.6):

$$
\begin{array}{rlrl}
\text { Case i : } & \Delta S(t) \propto t^{\frac{\sqrt{\xi(d-1)}}{\kappa}}+\mathcal{O}\left(N^{-2}\right), & & t \rightarrow 0^{+} \\
\text {Case ii : } & \Delta S(t) \propto e^{-C^{\prime} t^{-\frac{1}{\alpha}}}+\mathcal{O}\left(N^{-2}\right), & t \rightarrow 0^{+},
\end{array}
$$

\footnotetext{
${ }^{22}$ One can address the same issue in the string frame. In this frame the curvature invariants vanish in the transition limit (see appendix E), however a large value of the dilaton signals breakdown of our assumption of weak gravitational interactions. In section 6 , we construct explicit examples and show that the condition derived here is essentially equivalent to choosing the boundary value of the dilaton small enough so that it stays small in the transition region.

${ }^{23}$ As discussed in section 2.1 , by vanishing of the entropy we really mean it becomes of $\mathcal{O}\left(N^{-2}\right)$ in the large $\mathrm{N}$ limit.
} 
where $C^{\prime}$ is defined below (4.8).

The specific heat is obtained from $\Delta C_{h}=T d \Delta S / d T$ as,

$$
\begin{array}{rlrl}
\text { Case i : } & \Delta C_{v}(t) \propto t^{\frac{\sqrt{\xi(d-1)}}{\kappa}-1}+\mathcal{O}\left(N^{-2}\right), & & t \rightarrow 0^{+} \\
\text {Case ii : } & \Delta C_{v}(t) \propto e^{-C^{\prime} t^{-\frac{1}{\alpha}}} t^{-\frac{1}{\alpha}-1}+\mathcal{O}\left(N^{-2}\right), & t \rightarrow 0^{+},
\end{array}
$$

Clearly, for any $\kappa>\sqrt{\xi(d-1)}$ the specific heat diverges at the transition. In the special case of (4.10) with $n=2$, it becomes discontinuous, as it should in a second order transition.

\subsection{Speed of sound}

The (normal) speed of sound is defined as $c_{s}^{2}=d P / d E$. In isotropic systems that we consider here, the pressure is given by the free energy density and the speed of sound in the $\mathrm{BH}$ phase is most easily determined from

$$
c_{s, B H}^{2}=\frac{S_{B H}}{C_{v, B H}} .
$$

Now we ask the question, what does $c_{s, B H}^{2}$ become in the limit $T \rightarrow T_{c}$ ? Equation (4.19) can be written as,

$$
c_{s, B H}^{2}=\frac{\Delta S+S_{T G}}{\Delta C_{v}+C_{v, T G}} .
$$

For the case of a 3rd or higher order transition, the leading order terms $\Delta S$ and $\Delta C_{v}$ vanish as in section 4.3 and one is left with the terms of $\mathcal{O}\left(N^{-2}\right)$ both in the numerator and the denominator. Their ratio is $\mathcal{O}(1)$ and it is given by $S_{T G} / C_{v, T G}$ which is nothing but the speed of sound in the thermal gas phase. Therefore the speed of sound stays continuous across the transition, which is indeed what should happen for a transition of order higher than 2. In the case of a second order transition $\Delta S$ vanishes, but $\Delta C_{v}$ stays constant and one finds a discontinuity in the speed of sound. All of this is completely in accord with a system that obeys the laws of thermodynamics. However there is an important ambiguity which we discuss below.

\subsection{An order of limits issue and divergence in the dilaton}

An ambiguity arises when we consider the $N \rightarrow \infty$ limit in the discussion above: We observed that taking the $t \rightarrow 0$ limit while keeping $N$ finite one obtains $c_{s}^{2} \sim \mathcal{O}(1)$ at the transition point. Now, one can further take the $N \rightarrow \infty$ limit and $c_{s}^{2}$ still stays the same. On the other hand, had we taken the $N \rightarrow \infty$ limit first, then the $\mathcal{O}\left(N^{-2}\right)$ terms in (4.20) would drop out and we would obtain the result $c_{s}^{2} \sim t$ around the transition. Thus, we learn that the limits $N \rightarrow \infty$ and $t \rightarrow 0$ do not commute.

The resolution of the puzzle is clear: one cannot really take the $t \rightarrow 0$ limit for finite $N$ without taking into account the gravitational corrections. This is because the Einstein's theory breaks down in this limit. For definiteness, let us consider Einstein's gravity as an effective theory to a $d+1$ dimensional non-critical string theory. ${ }^{24}$ Then, the effective

\footnotetext{
${ }^{24}$ The precise nature of this theory is irrelevant for the sake of discussion. One could also consider a compactification of II(A)B on a $9-d$ dimensional internal manifold.
} 
Newton's constant at point $r$ is given by

$$
G_{\text {eff }}=\left(16 \pi M_{p}^{d-1} N^{2}\right)^{-1} e^{2 \Phi(r)} \sim g_{s}^{2} \ell_{s}^{d-1} e^{2 \Phi(r)},
$$

where $g_{s}$ is the value of the dilaton field on the boundary. To be definite, we consider an asymptotically AdS geometry where the dilaton approaches a constant value $\Phi(r) \rightarrow \Phi_{0}$ as $r \rightarrow 0$ and $g_{s}=\exp \left(\Phi_{0}\right)$. This implies that, for finite $\mathrm{N}$ there is a limit in $r_{h}$ beyond which we cannot ignore the gravitational interactions. In the specific case of (3.1) it is determined from (4.21) as ${ }^{25}$

$$
t_{l i m} \sim C\left(4 \sqrt{\pi}\left(M_{p} \ell\right)^{(d-1) / 2} N\right)^{-\kappa},
$$

where $C$ is the constant defined in (3.1). Beyond this limit one should take into account the gravitational corrections.

One can ask whether one can send $t_{l i m}$ to zero by fine-tuning a parameter in the theory. One can indeed fine-tune $t_{\lim }$ to be arbitrarily small by choosing $C$ or $1 / N^{2} \sim e^{\Phi_{0}}$ to be small. Sending $C$ to zero is not desirable, as in this case the subleading term in (2.22) vanishes and the resulting potential displays no transition. But one can send $e^{\Phi_{0}}$ to zero instead. This corresponds to the strict $N \rightarrow \infty$ limit.

The conclusion is that for a system that corresponds to finite $N$, regardless how large $\mathrm{N}$ is, one should consider $\mathcal{O}\left(G_{D}\right)$ corrections in order to calculate the value of a quantity such as $c_{s}^{2}$. On the other hand, the expected result that $c_{s}^{2}$ becomes discontinuous at $T_{c}$ in a second order and continuous in a higher order transition should still follow after taking into account the $\mathcal{O}\left(G_{D}\right)$ corrections because $\Delta S$ and $\Delta C_{c}$ should still vanish at $T_{c}$ also after the corrections. The only ambiguity is in determining the value of a quantity given by a ratio such as $c_{s}^{2}$, precisely at $T_{c}$. We encounter other examples of such quantities below.

\section{$5 \quad$ Viscosities and dispersion}

Viscosity coefficients are important observables probing the dissipative behavior in the dual field theory at finite temperature. In our case there are only two viscosity coefficients, that determine the response of the system to shear and bulk deformations respectively.

\subsection{Shear deformations}

The shear viscosity is given on the gravity side by the flux of the "shear" gravitons, i.e. the traceless-transverse component $h_{T T}$ of the metric fluctuations. Due to the well-established universality in the case of two-derivtive gravity $[20,21]$ the shear viscosity to entropy ratio in the $\mathrm{BH}$ phase is given by,

$$
\frac{\eta}{S}=\frac{1}{4 \pi}+\cdots
$$

where the ellipsis refer to $\alpha^{\prime}$ and $1 / N$ corrections. We note that at the classical level in the two-derivative Einstein-scalar gravity no temperature dependence arises in this ratio, and the result is simply given by (5.1) for at any $T \neq T_{c}$.

\footnotetext{
${ }^{25}$ we considered the dimensionless effective Newton's constant $G_{\mathrm{eff}} \ell^{d-1}$.
} 
What happens exactly at $T=T_{c}$ is similar to the story of the speed of sound above. To be more precise by including the $1 / N^{2}$ corrections, one should write $\eta=\eta_{0}+\delta \eta$ where $\eta_{0}$ is the leading order piece in the $1 / N^{2}$ expansion, $\eta_{0}=\Delta S / 4 \pi$ and $\delta \eta$ refers to $1 / N^{2}$ corrections. One has,

$$
\frac{\eta}{S_{B H}}=\frac{\eta_{0}+\delta \eta}{\Delta S+S_{T G}}=\frac{1}{4 \pi}+\cdots
$$

In the limit $T \rightarrow T_{c}$ the leading order terms vanish, but one still obtains a $\mathcal{O}\left(N^{0}\right)$ answer,

$$
\frac{\eta}{S_{B H}} \rightarrow \frac{\delta \eta}{S_{T G}}
$$

In order to determine the value of this, one has to include the $\mathcal{O}\left(1 / N^{2}\right)$ corrections in the analysis. The situation is different in the strict $N \rightarrow \infty$ limit where the $\mathcal{O}\left(N^{-2}\right)$ pieces in (5.2) drop out and one retrieves the universal value $\eta / s=1 / 4 \pi$ also at the transition point.

\subsection{Bulk deformations}

The bulk viscosity on the other hand is more interesting as it displays $\mathrm{T}$ dependence already at the two-derivative level $[22,23]$. It is given on the gravity side, by the flux of the rotationally-invariant fluctuations of the metric $h_{b} \equiv h_{11}=h_{22}=\cdots=h_{d-1, d-1}$. The computation is much more involved than the shear case, due to mixing of these fluctuations with the fluctuations of the scalar field $\delta \phi$. An ingenious method to disentangle this mixing is devised by Gubser et al in [22]. The main idea is to consider a gauge where the fluctuations of the scalar is set to zero. This is equivalent to carrying out the computation by using $\phi$ as the radial variable. The method is described in detail in [22] for the case of $d=4$. Here, we generalize this calculation to arbitrary dimensions. This is straightforward, yet technically involved and we present the details of this computation in appendix F . We shall only highlight the main steps and results here.

Kubo's linear response theory [24] relate the bulk viscosity to the retarded Green function of the stress tensor as ${ }^{26}$

$$
\zeta_{0}=-\frac{4}{(d-1)^{2}} \lim _{\omega \rightarrow 0} \frac{1}{\omega} \operatorname{Im} G_{R}(\omega)
$$

where the Green function is evaluated at zero momentum and it is defined by,

$$
G_{R}(\omega)=-i \int d t d^{d-1} x e^{i \omega t} \theta(t)\left\langle\left[\frac{1}{2} T_{i}^{i}(t, \vec{x}), \frac{1}{2} T_{j}^{j}(t, \vec{x})\right]\right\rangle,
$$

and $i, j$ denote the spatial components only. The imaginary part of the Green function maps to the flux of the $h_{b}$ gravitons on the gravity side. It is determined by a long and non-trivial computation (cf. [22] and appendix F of this paper). The computation is carried out in the gauge $\delta \Phi=0$. In other words $\Phi$ is taken as the radial variable. We denote the metric functions in this gauge as,

$$
d s^{2}=e^{2 A(\Phi)}\left(-f(\Phi) d t^{2}+d x_{d-1}^{2}\right)+e^{2 B(\Phi)} \frac{d \Phi^{2}}{f(\Phi)} .
$$

\footnotetext{
${ }^{26}$ The subscript 0 refers to the leading term of the quantity in the $1 / N^{2}$ expansion.
} 
The final result reads,

$$
\operatorname{Im} G_{R}(\omega)=-\frac{\mathcal{F}}{16 \pi G_{D}}=-\left.i \frac{\xi}{64 \pi G_{D}} \frac{f}{A^{\prime 2}} e^{(d-2) A-B}\left(h_{b}^{*} h_{b}^{\prime}-h_{b} h_{b}^{*^{\prime}}\right)\right|_{\Phi_{h}}
$$

where prime denotes $d / d \Phi$. The metric fluctuation $h_{b}$ satisfies the following second order equation:

$$
h_{b}^{\prime \prime}+h_{b}^{\prime}\left(\frac{2 \xi}{d-1} \frac{1}{A^{\prime}}+(d-2) A^{\prime}-3 B^{\prime}+\frac{f^{\prime}}{f}\right)+h_{b}\left(\frac{\omega^{2}}{f^{2}} e^{2 B-2 A}+\frac{f^{\prime}}{f} B^{\prime}-\frac{\xi}{d-1} \frac{f^{\prime}}{f A^{\prime}}\right)=0 .
$$

Here prime denotes $d / d \Phi$. In passing we note that this equation looks simpler and more transparent in the $r$-variable:

$$
\ddot{h}_{b}+\dot{h}_{b}\left((d-1) \dot{A}+\frac{\dot{f}}{f}+2 \frac{\dot{X}}{X}\right)+h_{b}\left(\frac{\omega^{2}}{f^{2}}-\frac{\dot{f}}{f} \frac{\dot{X}}{X}\right)=0 .
$$

where dot denotes $d / d r$ now. We note that reference to the normalization of the kinetic term $\xi$ disappears in this form. We also note that it reduces to the shear fluctuation equation for $X=$ const.

Equation (5.9) is solved by the boundary conditions $h_{b}=1$ on the boundary and the in-falling boundary condition at the horizon:

$$
h_{b} \rightarrow c_{b}\left(r_{h}-r\right)^{-i \frac{\omega}{4 \pi T}}, \quad r \rightarrow r_{h}
$$

Here $c_{b}$ is a T-dependent multiplicative factor. Inserting (5.10) in (5.7) (and changing variables to $\Phi$ ) yield,

$$
\operatorname{Im} G_{R}(\omega)=-\frac{\mathcal{F}}{16 \pi G_{D}}=\left.\frac{\omega(d-1)^{2}}{32 \pi \xi} S_{B H}\left|c_{b}\right|^{2}\left(\frac{V^{\prime 2}}{V^{2}}\right)\right|_{\Phi_{h}}
$$

Now, the bulk viscosity follows from the Kubo's formula (5.4)

$$
\frac{\zeta_{0}}{S_{B H}}=\left.\frac{1}{8 \pi \xi}\left|c_{b}\right|^{2}\left(\frac{V^{\prime 2}}{V^{2}}\right)\right|_{\Phi_{h}} .
$$

\subsection{Universality at $T_{c}$}

In the limit $\Phi_{h} \rightarrow \infty$ i.e. $T \rightarrow T_{c}$ one obtains a universal result for the bulk-viscosityto-entropy ratio. As shown in [23] the coefficient $\left|c_{b}\right|$ approaches to 1 in that limit. The ratio $V^{\prime} / V$ also becomes a constant that is given by the exponent in equation (2.22). Thus, (5.12) becomes,

$$
\left.\frac{\zeta_{0}}{S_{B H}}\right|_{T_{c}}=\frac{1}{2 \pi(d-1)},
$$

at the transition. We note that this is a universal result in the sense that it depends neither on the value of $T_{c}$ nor on the details of the underlying field theory system, nor on the order of the phase transition. The details of the computation that leads to this result are presented in App F. 
As for the shear case, the result is valid only in the limit where one can ignore gravitational interactions. Just as in the discussion below equation (5.1) above, if there exists $1 / N^{2}$ corrections then the result changes not by $\mathcal{O}\left(N^{-2}\right)$ but by $\mathcal{O}\left(N^{0}\right)$. Denoting the $1 / N^{2}$ correction to $\zeta_{0}$ as $\delta \zeta$, one has,

$$
\frac{\zeta}{S_{B H}}=\frac{\zeta_{0}+\delta \zeta}{\Delta S+S_{T G}} \rightarrow \frac{\delta \zeta}{S_{T G}}, \quad \text { as } T \rightarrow T_{c} .
$$

The latter is determined by computing the $1 / N^{2}$ corrections in the background and there is no reason that it should coincide with (5.13), even in the large $N$ limit. However if one considers a system with $N=\infty$ strictly, then one should take the $N \rightarrow \infty$ before $t \rightarrow 0$ and the result can be trusted in principle. In the next section we construct explicit kink solutions that flow from an AdS on the boundary (with a fixed value of $e^{\Phi_{0}}$ ) and lineardilaton in the deep interior. In these solutions one can indeed send $e^{\Phi_{0}} \rightarrow 0$ in a consistent fashion and one obtains the universal result above. It would be extremely interesting to find a similar solution in IIB and check whether the result given by (5.13) holds there.

\subsection{Scaling of viscosities near $T_{c}$}

There is no ambiguity however, in the scaling of the viscosities $\eta$ and $\zeta$ near $T_{c}$, as this is determined by the leading piece in the $1 / N^{2}$ expansion. From the results above, we find that both $\eta$ and $\zeta$ scales as the entropy density near $T_{c}$ :

$$
\eta, \zeta \propto \Delta S(t) \propto e^{(d-1) A(t)} .
$$

The latter is given by (4.5) and (4.6) in cases i and ii:

$$
\begin{array}{cll}
\text { Case i : } & \eta, \zeta \propto t^{\frac{\sqrt{\xi(d-1)}}{\kappa}} & t \rightarrow 0 \\
\text { Case ii : } & \eta, \zeta \propto e^{C^{\prime} t^{-\frac{1}{\alpha}}}, & t \rightarrow 0,
\end{array}
$$

where $C^{\prime}$ is a constant given below equation (4.8). The latter is the typical scaling of the order parameters in the Kosterlitz-Thouless transition [12, 13, 25].

\subsection{Dispersion relation of pressure waves}

Now, we are in a position to write down the dispersion relation of pressure waves near criticality, including dissipative terms. The hydrodynamics predict a dispersion relation of the form,

$$
\omega=c_{s} \mathbf{q}-\frac{i}{T}\left(\frac{d}{d-1} \frac{\eta}{S_{B H}}+\frac{1}{2} \frac{\zeta}{S_{B H}}\right) \mathbf{q}^{2}+\cdots
$$

From the results in the previous two subsections, eqs. (5.1) and (5.13), in the strict $N=\infty$ limit one finds,

$$
\begin{array}{rlr}
\text { Case i : } & \omega \rightarrow c_{s} \mathbf{q}-\frac{i}{4 \pi T_{c}}\left(\frac{d+1}{d-1}\right) \mathbf{q}^{2}, & t \rightarrow 0 \\
\text { Case ii }: & \omega \rightarrow c_{s} \mathbf{q}-\frac{i}{4 \pi T_{c}}\left(\frac{d+1}{d-1}\right) \mathbf{q}^{2}, & t \rightarrow 0,
\end{array}
$$

where we only show the leading terms in $t$ in both of the terms. One should remember the ambiguities in these formula for finite $\mathrm{N}$ as explained in the previous subsection. 


\section{$6 \quad$ UV geometry and analytic kink solutions}

So far we have not specified the geometry near the boundary. The only assumption we made in the derivation of section 2 is that $A^{\prime}(r)<0$ near $r \approx 0$. This includes the case of asymptotically AdS backgrounds. In fact, for holographic applications this is the most desirable case. Therefore it would be interesting to find explicit solutions that flow from an AdS geometry in the UV to a geometry of the type given by the solution of the IR potential (2.22) with $V_{\text {sub }}$ given by either of (3.1) or (3.2). In this section we construct such explicit examples for the case of (3.1). This will the most interesting case for applications to holographic superfluids in [11].

An asymptotic AdS geometry is also desirable for another reason. In the case when the scalar can be regarded as the dilaton of NCST, its value goes over to a constant $\Phi(0)=\Phi_{0}$ in an asymptotic AdS geometry which provides a tunable parameter. On the other hand, the effective Newton's constant is related to this value as,

$$
G_{D} \propto e^{2 \Phi_{0}} .
$$

Therefore, this value can be tuned to make the gravitational interactions very small. In other words, $\Phi_{0}$ will be the parameter that corresponds to the "color" $\mathrm{N}$ in a dual field theory

$$
N^{2} \propto e^{-2 \Phi_{0}} .
$$

The large $\mathrm{N}$ limit corresponds to tuning $\Phi_{0}$ to be very large.

At the same time, we argued in section 4.2 that in order to keep the transition region away from the curvature singularity, one needs another fine tuning $C \ll 1$ where $C$ is the constant defined in (3.1). In principle, these two separate fine-tunings may contradict each other. Here, we will show by constructing explicit examples that, on the contrary, these two fine-tunings are essentially the same. ${ }^{27}$ We shall focus on the case where the scalar field is regarded as the dilaton and its normalization in (2.1) is given by $\xi=4 /(d-1)$.

\subsection{Two-exponent potentials}

For notational simplicity we define the function $\lambda$

$$
\lambda \equiv e^{\Phi}, \quad \lambda_{0} \equiv e^{\Phi_{0}} .
$$

Consider the potential,

$$
V(\lambda)=V_{\infty} \lambda^{\frac{4}{d-1}}\left(1+C \lambda^{-\kappa}\right) .
$$

According to the discussion in section 3, the black-hole solution that follows from this potential exhibits a continuous type Hawking-Page transition with the order of the transition determined by the constant $\kappa$ as in (4.10). For example the transition is second order for $\kappa=2$. The transition region is in the asymptotic IR region $\lambda_{h} \gg 1$.

\footnotetext{
${ }^{27}$ This is not hard to see directly from (3.1) where shifting the vev of $\Phi$ can be regarded as tuning the constant $C$.
} 
We observe that this simple potential allows for an AdS extremum at ${ }^{28}$

$$
\lambda_{0}=C^{\frac{1}{\kappa}}\left(\frac{\kappa(d-1)}{4}-1\right)^{\frac{1}{\kappa}}
$$

when $\kappa(d-1)>4$. As a result, the two-exponent potential (6.4) should have a kink solution that flows from an asymptotically AdS geometry in the $U V$ at $\lambda=\lambda_{0}$ towards the asymptotically linear-dilaton geometry in the IR at $\lambda=\infty$.

We observe from (6.5) that the large $\mathrm{N}$ limit (6.2) corresponds to $C \ll 1$. This is reassuring because the same limit is required in section 4.2 in order to separate the large curvature region from the transition region.

One can construct this solution analytically for the thermal gas case (2.2), for special values of $\kappa$ and $d$. This can easily be done by the method of scalar variables, appendix $\mathrm{A}^{29}$ as follows: The TG solution corresponds to the special solution of (3.10) $Y=0$. In this case the Einstein-dilaton system reduces to a single first order differential equation,

$$
\frac{d X_{0}}{d \Phi}=-\gamma\left(1-X_{0}^{2}\right)\left(1+\frac{1}{2 \gamma} \frac{1}{X_{0}} \frac{d \log V}{d \Phi}\right)
$$

where $\gamma$ is given by (3.8),

$$
\gamma=\frac{2 \sqrt{d}}{d-1}
$$

A very nice coincidence is that one can construct an analytic solution to (6.6) precisely in the most interesting case, namely $\kappa=2, d-1=3$. This case corresponds to a second order phase transitions in three spatial dimensions, indeed the most relevant case for many condensed matter applications! [11]. The solution reads,

$$
X_{0}(\lambda)=-\frac{1}{2} \frac{\lambda^{2}-\lambda_{0}^{2}}{\lambda^{2}+\frac{1}{2} \lambda_{0}^{2}} .
$$

In the UV limit $X_{0} \rightarrow 0$ which indeed corresponds to an asymptotically AdS geometry. ${ }^{30}$ To see this, one recalls the definition of $X_{0}$ in (3.7) and vanishing $X$ corresponds to constant dilaton.

One can construct the metric and the dilaton in the r-frame explicitly by solving equations (A.5) and (A.6) with $X=X_{0}$ given by (6.8) and $Y=0$. The result is,

$$
\begin{aligned}
& d s^{2}=\lambda_{0}^{-\frac{4}{3}} \frac{\operatorname{ch}^{\frac{2}{3}}\left(\frac{3 r}{2 \ell}\right)}{\operatorname{sh}^{2}\left(\frac{3 r}{2 \ell}\right)}\left(d t^{2}+d x_{d-1}^{2}+d r^{2}\right), \\
& \lambda(r)=\lambda_{0} \operatorname{ch}\left(\frac{3 r}{2 \ell}\right) .
\end{aligned}
$$

\footnotetext{
${ }^{28}$ We assume $C>0$.

${ }^{29}$ It can also be obtained by the method of "fake super-potential", [26].

${ }^{30}$ Equation (6.6) looks singular in the limit $X_{0} \rightarrow 0$. In fact it is not as the pole of $X_{0}$ at $\lambda_{0}$ is cancelled against the pole in $V^{\prime}(\lambda)$
} 
Clearly, the geometry becomes AdS near $r=0$. The metric in the string-frame is obtained from (6.9) by the Weyl transformation $d s_{s t}^{2}=\lambda^{4 / 3} d s^{2}$ :

$$
d s_{s t}^{2}=\operatorname{coth}^{2}\left(\frac{3 r}{2 \ell}\right)\left(d t^{2}+d x_{d-1}^{2}+d r^{2}\right) .
$$

In the IR region $r \rightarrow \infty$ the background in the string-frame becomes linear-dilaton:

$$
d s_{s t}^{2} \approx d t^{2}+d x_{d-1}^{2}+d r^{2}, \quad \Phi(r) \approx \frac{3 r}{2 \ell} .
$$

Therefore the background (6.11) and (6.10) is a solution to the 5D Einstein-dilaton theory that interpolates between an AdS background near the boundary and a linear-dilaton background in the deep interior in the string frame.

It would be very useful to obtain an analytic black-hole solution to the potential (6.4) by turning on a blackness function in (6.9). In the language of scalar variables (3.9), (3.10), this corresponds to turning on a non-trivial $Y$. Now, the system becomes more complicated and unfortunately we could not find an analytic solution. However a numerical solution can easily be constructed by solving the system by a numerical code. We checked that the black-hole obtained this way obeys the general conditions described in section 2.2, in particular it asymptotes the TG kink above in the limit $r_{h} \rightarrow \infty$. To our knowledge, the closest analytic BH solutions are the Chamblin-Reall solutions to single exponential potentials [27]. However, these solutions do not exhibit any Hawking-Page transitions.

\subsection{Three-exponent potentials}

The analytic solution found above is for the particular case $\kappa=2, d-1=3$. It is interesting to search for similar analytic solutions for an arbitrary $\chi$ and $d$. This is not possible with a simple two-exponential dilaton potential. Instead we consider a three-exponential potential of the form:

$$
V(\lambda)=V_{\infty} \lambda^{\frac{4}{d-1}}\left(1+C \lambda^{-\kappa}+\tilde{C} \lambda^{-2 \kappa}\right) .
$$

This potential possesses AdS extrema for generic values of $\kappa$ and $d$. Let us again consider a thermal-gas type 2.2 ansatz. One can find an analytic solution for the special value of $\tilde{C}$ :

$$
\tilde{C}=\frac{C^{2}}{(\kappa+2)^{2}}\left(1+\kappa-\frac{\kappa^{2}}{4}(d-1)\right) .
$$

In fact this class of potentials can be regarded as a generalization of (6.4) with $\kappa=2$ and $d-1=3$. In this special case the constant $\tilde{C}$ in (6.14) vanishes and the three-exponential potential (6.13) reduces to (6.4). Solution of (6.6) in the general case reads,

$$
X_{0}(\lambda)=-\frac{1}{\sqrt{d}} \frac{\lambda^{\kappa}-\lambda_{0}^{\kappa}}{\lambda^{\kappa}+\frac{1}{q} \lambda_{0}^{2}}, \quad q \equiv \frac{\kappa}{2}(d-1)-1 .
$$

One checks that, this solution reduces to (6.8) in the special case of $\kappa=2, d-1=3$. The special point $\lambda_{0}$ corresponds to the extremum of (6.13) and it is given by,

$$
\lambda_{0}=\left(\frac{C q}{\kappa+2}\right)^{\frac{1}{\kappa}} \text {. }
$$


The solution (6.15) corresponds to a kink that interpolates between an AdS geometry at $\lambda_{0}$ to an asymptotically linear-dilaton background at $\lambda \rightarrow \infty$.

We again observe that by tuning the constant $C$ to small values one can establish two goals at the same time, namely to separate the transition region $t \approx 0$ and the high curvature region $\lambda_{h} \gg 1$ and to make the effective gravitational coupling weak.

It is not possible to obtain a closed form expression for the metric in the $\mathrm{r}$-frame ${ }^{31}$ for generic $\kappa$ and $d$ but it is easy to write down the metric directly in the $\lambda$-frame:

$$
d s^{2}=\lambda^{-\frac{4}{(d-1)}}\left(1-\frac{\lambda_{0}^{\kappa}}{\lambda^{\kappa}}\right)^{-\frac{2}{q}}\left[d t^{2}+d x_{d-1}^{2}+c_{q}\left(1-\frac{\lambda_{0}^{\kappa}}{\lambda^{\kappa}}\right)^{\frac{2}{q}-2} \lambda^{-2} d \lambda^{2}\right]
$$

where $q$ is given in terms of $\kappa$ and $d$ in (6.15) and the constant $c_{q}$ is given by,

$$
c_{q}=\left(\frac{2 \ell}{q(d-1)}\right)^{2}
$$

The metric is defined in the range $\lambda \in\left(\lambda_{0}, \infty\right)$. It is easy to see that the metric becomes AdS near $\lambda \approx \lambda_{0}$ by a change of variable $\lambda-\lambda_{0} \propto r^{q}$ near $r \approx 0$. On the other hand, for large values of $\lambda$ it asymptotes to the linear-dilaton background in the string frame:

$$
d s_{s t}^{2} \approx d t^{2}+d x_{d-1}^{2}+\left(\frac{2 \ell}{q(d-1)}\right)^{2} d \Phi^{2} .
$$

The solution given by (6.15) constitutes a one-parameter family of kink solutions parametrized by $\kappa$ - that interpolates between AdS and linear-dilaton backgrounds in the string-frame. The geometry is regular everywhere in the string frame. It would be very interesting to embed this geometry in IIB. One may be able to do so, by finding an analogous solution in a consistent truncation of IIB with non-trivial dilaton. Apart from our main interest in this work, namely that black-holes on these backgrounds exhibit continuous type Hawking-Page transitions with the order parametrized by $\kappa$, they may have other applications in different contexts.

\section{$7 \quad$ Stringy corrections}

In this paper, we consider two-derivative Einstein-scalar gravity in the limit of small gravitational corrections, $G_{D} \rightarrow 0$. On the other hand, we showed that the continuous phase change occurs in a singular limit, where the black-hole horizon marginally traps a curvature singularity. A crucial issue then, is the possible $\alpha^{\prime}$ or $G_{D}$ corrections at this point. We comment on these issues in this section.

First of all, we should stress that the results that are obtained above are consistent and robust in the framework of Einstein's gravity. The two-derivative theory breaks down only near curvature singularities, or when the dilaton field becomes very large. On the other hand the curvature singularity is always cloaked by the BH horizon as one approaches the transition point $t \rightarrow 0^{+}$, and the dilaton can always be kept from growing too large as

\footnotetext{
${ }^{31}$ At best one can express $r$ in terms of $\lambda$ as a combination of hypergeometric functions.
} 
long as $t \neq 0$ strictly by fine-tuning the dilaton potential. How to manage this is explained in section 4.2. Therefore the questions, as well as the answers that are studied so far are definite within this framework.

On the other hand, a basic motivation for us to study continuous phase transitions in gravity concerns possible applications in the dual gauge theories or condensed matter systems through the holographic correspondence, and a robust framework to relate the two sides of the correspondence is string theory rather than Einstein's gravity. Therefore, one should consider possible stringy corrections if one desires to interpret the results of this paper from an holographic point of view.

\subsection{Higher derivative corrections}

The stringy higher derivative corrections to Einstein's gravity are measured by the typical curvature of the geometry $R \ell_{s}^{2}$ in units of string length $\ell_{s}=\sqrt{\alpha^{\prime}}$. Concerning these corrections, one can ask two separate questions:

1. Are the conditions derived in sections 2.2 and 3 for the existence and the type of the continuous transitions still valid once we include the $\alpha^{\prime}$ corrections?

2. For which quantities and in which regions can we trust the observables if we ignore the $\alpha^{\prime}$ corrections?

Let us consider the first question above. For existence of a continuous phase transition, clearly, the ( $\alpha^{\prime}$ corrected) geometries should asymptotically become the same in the IR region. For a $\mathrm{BH}$ geometry that asymptotes a TG geometry near the boundary, this condition can most generally expressed as vanishing of the ADM mass of the BH.

Let us now specify to the case when the scalar is the dilaton field of the $d+1$ dimensional non-critical string theory. Then the normalization of the kinetic term is given by (2.25). The required asymptotics for a continuous phase transition is given by $(2.26)$ :

$$
V \rightarrow e^{\frac{4}{d-1} \Phi}
$$

In the string frame $g_{s, \mu \nu}=\exp (2 \Phi / d-1) g_{\mu \nu}$ the action reads

$$
\mathcal{A}_{s}=\frac{1}{16 \pi G_{D}} \int d^{d+1} x \sqrt{-g_{s}} e^{-2 \Phi}\left(R_{s}-\xi_{s}(\partial \Phi)^{2}+V_{s}(\Phi)+\cdots\right),
$$

where the subscript s refer to string frame quantities and the ellipsis denote the (unknown) higher derivative corrections. Here the string frame potential is related to the Einstein frame potential by,

$$
V(\Phi)=V_{s}(\Phi) e^{\frac{4}{d-1} \Phi}
$$

Therefore the requirement for existence of a continuous transition now becomes $V_{s} \rightarrow$ const. in (7.1), as $\Phi \rightarrow \infty$. The remarkable fact is that, the unique solution to this asymptotic potential with the TG ansatz (or with the $\mathrm{BH}$ ansatz in the limit $\Phi_{h} \gg 1$ ) is nothing else but the linear-dilaton solution, that is $\alpha^{\prime}$ exact! Therefore, we expect that the condition (2.22) be $\alpha^{\prime}$ exact in this case. 
There is still a possibility of $\alpha^{\prime}$ corrections in the sub-leading terms (3.1) and (3.2). This means that the constants $\kappa$ and $\alpha$ in these equations can get renormalized by the $\alpha^{\prime}$ corrections. $^{32}$ However, clearly this would only affect the definition of these constants. In other words, an nth order transition would be given by $\kappa=2 /(n-1)+\mathcal{O}\left(\alpha^{\prime}\right)$ instead of (4.10).

Finally, in the case when the scalar is not the dilaton of a non-critical string, then there is a possibility that also the leading term (2.22) be corrected. Avoiding this is another benefit of identifying the scalar with the dilaton of $d+1$ dimensional non-critical string theory.

Now we move on to the second question above. First consider the thermodynamic functions: The entropy of the black-hole should receive the usual $\alpha^{\prime}$ corrections. These are of two different origin: a) The definition of the entropy as the horizon size will be corrected by Wald's generalization [28]; b) the background geometry itself will receive $\alpha^{\prime}$ corrections. The definition of the surface gravity, hence the temperature, is subject to similar corrections of two separate origin. However, the first law of thermodynamics should still be valid, therefore we expect that the equation (3.17) (or (2.19)) should survive the corrections. Clearly, all of the other thermodynamic quantities that we obtained in section 4 are subject to $\alpha^{\prime}$ corrections in the region of high curvature in the Einstein frame. We expect these corrections to renormalize constants $\kappa$ and $\alpha$ in (3.1) and (3.2).

Secondly, one can ask if the thermodynamic quantities are subject to higher derivative corrections away from the transition region. The size of the corrections away from the singularity should be determined by the ratio $\ell_{s} / \ell$ where $\ell$ is the typical size of the asymptotic geometry, e.g. it is the AdS radius, if the near boundary geometry is asymptotically AdS. This ratio is holographically related to an observable in the dual field theory. For instance, it is proportional to the gauge string tension in the holographic QCD models [8], whereas it is related to the interaction strength between the spins in spin-models [11]. Then, the issue of corrections to thermodynamic quantities become a phenomenological question, and the answer depends on the particular dual field theory that one desire to describe holographically. For example, in the holographic QCD models considered in [8], it was found by matching the string tension on the lattice-QCD that the ratio is $\mathcal{O}\left(10^{-1}\right)$ and the $\alpha^{\prime}$ corrections are small.

Finally, we note that the quantities that are naturally evaluated in the string frame should be trustable in the vicinity of the transition because the curvature invariants in the string frame in the transition region becomes very small as shown in appendix E. Such quantities involve F-string and $D$-brane configurations in the probe approximation. In the companion paper, [11], we map these configurations to spin-correlation functions in a dual spin-model. Therefore, we expect that these important observables should be immune to $\alpha^{\prime}$ corrections - except that corrections may arise through dependence on the scaling constants $\kappa$ and $\alpha$ in (3.1) and (3.2). We refer the reader to [11] for a more detailed discussion.

\footnotetext{
${ }^{32}$ There is also the possibility of power-law corrections in (3.1) and log-corrections in (3.2)
} 


\section{$7.21 / N^{2}$ corrections}

Similarly the gravitational interactions for finite $G_{D}$ also becomes important near the transition region $t \sim 0$. This is because the dilaton field ${ }^{33}$ diverges precisely at $t=0$. This means that the effective string coupling $g_{s}$ becomes large in this region, unless $N$ can be set to $\infty$ in a strict manner.

If in the dual field theory one has finite $N$ - regardless how large it is - our calculations can only be trusted in the region

$$
t>C\left(4 \sqrt{\pi}\left(M_{p} \ell\right)^{(d-1) / 2} N\right)^{-\kappa} .
$$

For a derivation of this bound, we refer to section 4.5. Beyond this point, one should take into account corrections. Therefore, in the case of large but finite $N$ (small but finite $G_{D}$ ), what one has to do in order to compute the rational quantities at $T_{c}$, is to compute the spectrum of fluctuations in the TG geometry, and from this compute the determinant correction in the saddle-point approximation, in the limit $T \rightarrow T_{c}$.

We avoided this problem here by tuning the boundary value of the dilaton $e^{\Phi_{0}} \propto N^{-1}$ to be parametrically small so that the restriction above is pushed towards $t \rightarrow 0$.

On the other hand, we may be interested in the strict $N=\infty$ case for a physical reason. For example, in [11] we propose that the strict $N=\infty$ case corresponds to the XY-spin models that constitute canonical models of superfluidity. Then, if we are interested in a holographic model for superfluidity, we can take the strict $N \rightarrow \infty$ limit. In this case one can extend the safe region up to $t \rightarrow 0^{+}$. However, there is an analogous problem where the UV region becomes highly curved.

\section{Discussion}

There are several possible directions one can improve on the results obtained here:

- Holographic implications in dual field theories.

Hawking-Page transitions in gravity generically correspond to confinementdeconfinement transitions in the dual gauge theory [2]. Therefore, the backgrounds presented here are natural candidates for continuous type confinement-deconfinement transitions. More interestingly, it is also possible to relate gravity to field theories that describe continuum limit of certain condensed matter systems such as the spin models. We refer the reader to our companion paper [11] for a detailed exploration of this latter application.

- Embedding in string theory

One of main results is that the continuous transition corresponds to the limit where the BH horizon marginally traps a curvature singularity where the Einstein's gravity becomes not trustable. We avoided this problem by fine-tuning the coefficient of the subleading term in the potential to be very small. This crude way serves our purpose

\footnotetext{
${ }^{33}$ One should consider the case when the scalar is not dilaton separately, see the discussion in section 8 .
} 
to determine the qualitative scaling behavior of observables in the model, but clearly it is desirable to obtain better control over the model for holographic applications. In order to resolve the singularity one may think of embedding the background in the full string theory. Indeed we showed that the deep-interior asymptotics of the $\mathrm{BH}$ geometry near the transition is a linear-dilaton geometry in the case when the scalar is a dilaton, which is an exact solution in the string frame, in all orders in $\alpha^{\prime}$. This observation should be relevant in embedding the set-up in the full string theory. However we need more than this because the full flow geometry is not $\alpha^{\prime}$ exact and there is no obvious way to study this solution in a systematic expansion in the full string theory, because the typical curvature scale in the geometry is $\ell / \ell_{s} \sim 1$. Therefore, a better strategy is to start with a consistent truncation of a critical string theory and directly search for continuous critical phenomena there. A helpful starting point for this investigation may be the work of $[29]{ }^{34}$

We also observe that the asymptotic form of the scalar potential in (2.22) and (3.1) is sum of exponentials that quite generically appears in consistent truncations of IIB and IIA critical string theory. We leave this investigation for future work.

- The case when the scalar is not dilaton

Considering the scalar field as dilaton has various advantages. First of all, if one would like to think of the framework as an approximation to non-critical string theory, ${ }^{35}$ then the most economical choice is to consider $\Phi$ as the dilaton field of NCST. Secondly, for this choice the IR asymptotics become the linear dilaton geometry that is known as a full solution to NCST. As we show in [11], another reason is that, in this case scaling of certain observables in the dual field theory, such as the magnetization if the dual theory is a spin-model, has expected scaling properties as a function of $t$. Yet, it would be interesting to investigate the cases when $\Phi$ is just a scalar field, and work out the implications in a hypothetical dual theory. It is also interesting to search for continuous Hawking-Page transitions when there are more than one scalars present.

- The specification of the UV geometry

In this work, we were mostly interested in scaling of thermodynamic observables near the transition that corresponds to the IR limit of the geometry. For the various applications in holography however, the entire background geometry is important. In order to be able to apply the usual prescription of AdS/CFT to compute correlation functions, etc. it is desirable to construct a solution that has the desired IR asymptotics (2.22) and an asymptotically AdS UV geometry at the same time. This is done in section 6. One particularly interesting future direction is to find analogous solutions in IIB.

\footnotetext{
${ }^{34}$ We thank Yaron Oz for pointing out the relevance of this work and fruitful discussions on this issue.

${ }^{35}$ This approximation is viable only if $\ell_{s} / \ell$ is small so that neglecting the higher derivative terms can be an approximation. As we mentioned several times this is not a tunable parameter in our set-up but rather should be determined phenomenologically by matching a corresponding quantity in the dual field theory. For example in certain models of holographic QCD [30] it turns out that this parameter is indeed small, about $1 / 7$.
} 
- The transition in the case $G_{D} \neq 0$

One can ask what happens if we turn on (small) gravitational interactions but ignore the higher derivative corrections. In this case the entropy of the BH solution (normalized by $1 / N^{2}$ ) is given by,

$$
S_{B H}=\frac{e^{(d-1) A\left(\Phi_{h}\right)}}{4 G_{D} N^{2}}+\delta S_{B H}
$$

where $\delta S$ is the correction that stems from the determinant around the saddle which is $\mathcal{O}\left(G_{D}\right)$ with this normalization. The thermal radiation has a single contribution of the same order $S_{T G}=\mathcal{O}\left(G_{D}\right)$. Therefore, the continuous transition would occur at $\Phi_{c}$ that is determined by

$$
\frac{e^{(d-1) A\left(\Phi_{c}\right)}}{4 G_{D} N^{2}}=S_{T G}-\delta S_{B H}
$$

There are two qualitatively distinct solutions to this equation. In the first case both sides of this equation may vanish. This is the same situation that we studied in this paper in the classical limit. If this is what happens then we conclude that the $G_{D}$ corrections do not help avoiding the singularity and the transition still happens at the singular point where the scale factor vanishes. However, there is a second possibility, that the r.h.s. is finite, hence the transition is moved away from the singular point by the $G_{D}$ corrections. In order to find which possibility is realized one should compute the spectrum of fluctuations around the TG and the BH saddles at a temperature determined by $\Phi_{h}$ and see if the difference in the entropy can be matched to the l.h.s. in (8.1) at a finite $\Phi_{c}$. One should also check that this $\Phi_{c}$ does not lie beyond the point where the Jeans instability destroys the TG saddle.

- Adding other fields

One can extend the analysis by including other fields in the action (2.1). A particularly interesting case to consider is multiple scalar fields. One can ask what conditions should be imposed on the scalar potential $V\left(\Phi_{1}, \Phi_{2}, \cdots\right)$ in order to obtain continuous Hawking-Page transitions.

\section{Acknowledgments}

It is a pleasure to thank Alex Buchel, Bernard de Wit, Elias Kiritsis, Yaron Oz, Marco Panero, Erik Plauschinn, Giuseppe Policastro, Henk Stoof and Stefan Vandoren for valuable discussions. The author is supported by the VIDI grant 016.069.313 from the Dutch Organization for Scientific Research (NWO). Some part of this work has been completed during the program "Integrability in String and Gauge Theories; AdS/CFT Duality and its Applications" in Nordita, Stockholm, June 2010 where I benefited from many useful discussions with other participants. 


\section{A The scalar variables and reduction of the Einstein-scalar system}

The scalar variables of the Einstein-scalar system is defined [7, 9] as, In case of a neutral black-hole in $d+1$ dimensions that is solution to (2.1), one only needs two such variables, that we call $X$ and $Y$ :

$$
X(\Phi) \equiv \frac{\gamma}{d} \frac{\Phi^{\prime}}{A^{\prime}}, \quad Y(\Phi) \equiv \frac{1}{d} \frac{g^{\prime}}{A^{\prime}} .
$$

where the function $g$ is defined as $g=\log f$ and the constant $\gamma$ is given by,

$$
\gamma=\sqrt{\frac{d \xi}{d-1}}
$$

These functions satisfy:

$$
\begin{aligned}
& \frac{d X}{d \Phi}=-\gamma\left(1-X^{2}+Y\right)\left(1+\frac{1}{2 \gamma} \frac{1}{X} \frac{d \log V}{d \Phi}\right), \\
& \frac{d Y}{d \Phi}=-\gamma\left(1-X^{2}+Y\right) \frac{Y}{X} .
\end{aligned}
$$

This second order system is sufficient to determine all of the thermodynamic properties (and dissipation) of the gravitational theory [9]. This is a reduction of the fifth order Einstein-scalar system to an equivalent second order system.

It is straightforward to show that these equations combined with the following three,

$$
\begin{aligned}
& \frac{d A}{d r}=-\frac{1}{\ell} e^{A_{0}} e^{-\gamma \int_{\Phi_{0}}^{\Phi}\left(X(t)-\frac{1}{d} X^{-1}(t)\right) d t}, \\
& \frac{d \Phi}{d r}=-\frac{1}{\ell} e^{A_{0}} \frac{d}{\gamma} X(\Phi) e^{-\gamma \int_{\Phi_{0}}^{\Phi}\left(X(t)-\frac{1}{d} X^{-1}(t)\right) d t}, \\
& \frac{d g}{d r}=-\frac{1}{\ell} e^{A_{0}} d Y(\Phi) e^{-\gamma \int_{\Phi_{0}}^{\Phi}\left(X(t)-\frac{1}{d} X^{-1}(t)\right) d t},
\end{aligned}
$$

solve the original Einstein equations. Here $\Phi_{0}$ is a cut-off near the boundary. One can also express $g$ and $A$ in terms of the scalar variables directly from the definitions (A.1):

$$
\begin{aligned}
& A(\Phi)=A_{0}+\frac{\gamma}{d} \int_{\Phi_{0}}^{\Phi} \frac{1}{X} d \tilde{\Phi} \\
& g(\Phi)=\gamma \int_{\Phi_{0}}^{\Phi} \frac{Y}{X} d \tilde{\Phi}
\end{aligned}
$$

Another useful equation relates the scalar potential to the scalar variables, that follows from (A.3):

$$
V(\Phi)=\frac{d(d-1)}{\ell^{2}}\left(1+Y-X^{2}\right) e^{-2 \gamma \int_{-\Phi_{0}}^{\Phi}\left(X(t)-\frac{Y(t)}{2 X(t)}\right) d t} .
$$

The precise form of the overall coefficient follows from inserting (A.5), (A.6) and (A.7) in the Einstein equation (2.15).

The zero T solution (thermal gas) corresponds to the $Y=0$ fixed point of eqs. (A.3) and (A.4):

$$
\frac{d X_{0}}{d \Phi}=-\gamma\left(1-X^{2}\right)\left(1+\frac{1}{2 \gamma} \frac{d \log V}{d \Phi}\right)
$$


The solution to this equation determines the zero $\mathrm{T}$ scalar variable $X_{0}$. In [9] it is shown that,

$$
\lim _{\Phi_{h} \rightarrow \infty} X(\Phi)=X_{0}(\Phi) .
$$

Equation (A.11) provides a different representation of the scalar potential:

$$
V(\Phi)=\frac{d(d-1)}{\ell^{2}}\left(1-X_{0}^{2}\right) e^{\left.-2 \gamma \int_{-\Phi_{0}}^{\Phi} X_{0}(t)\right) d t} .
$$

\section{B Derivation of thermodynamics from the scalar variables}

The thermodynamics of the black-hole can directly be determined from the solution in terms of the scalar variables. The free energy is given by

$$
F\left(\Phi_{h}\right)=\frac{1}{4 G_{D}} \int_{\Phi_{h}}^{\infty} d \tilde{\Phi}_{h} e^{(d-1) A\left(\Phi_{h}\right)} \frac{d T}{d \tilde{\Phi}_{h}} .
$$

Our backgrounds satisfy the 1st law of thermodynamics $S=-d F / d T$. Equation (3.17) directly follows from integrating this equation by noting the equation (2.11)

$$
S=4 \pi e^{(d-1) A\left(r_{h}\right)}+\mathcal{O}\left(N^{-2}\right),
$$

and ignoring the $1 / N^{2}$ corrections. The temperature in the scalar variable systems is obtained from

$$
T\left(\Phi_{h}\right)=\frac{\ell}{4 \pi(d-1)} e^{A\left(\Phi_{h}\right)} V\left(\Phi_{h}\right) e^{\gamma \int_{\Phi_{0}}^{\Phi_{h}} X(\Phi) d \Phi} .
$$

Derivation of this equation is more involved. It follows from the generalization to arbitrary dimensions, of the "useful relation" equation (7.38) of [9]. The computation is analogous to the appendix H.11 of [9] with slight changes due to different multiplicative factors in the equations in appendix (A) above. One finds the generalized "useful relation" as:

$$
\frac{S}{T^{d-1}}=\frac{1}{4 G_{D}}\left(\frac{4 \pi(d-1)}{\ell}\right)^{d-1} \frac{e^{-\gamma(d-1) \int_{-\infty}^{\Phi_{h}} X}}{V\left(\Phi_{h}\right)^{d-1}},
$$

from which (B.3) follows immediately by use of (B.2).

\section{Sub-leading corrections to the geometry near $T_{c}$}

The sub-leading corrections are found by expanding the scalar variables as

$$
X(\Phi)=-\frac{1}{\sqrt{d}}+\delta X(\Phi), \quad Y(\Phi)=Y_{0}(\Phi)+\delta Y(\Phi)
$$

and inserting in (3.9) and (3.10). Then one obtains a linear differential equation for $\delta X$ that is solved by fixing the integration constant with the requirement of no singularity at $\Phi_{h}$ : One finds,

$$
\delta X(\Phi)= \begin{cases}\frac{\tilde{C} \kappa}{\kappa+\tilde{\gamma}} e^{-\kappa \Phi} \frac{1-e^{-(\kappa+\tilde{\gamma})\left(\Phi_{h}-\Phi\right)}}{1-e^{-\tilde{\gamma}\left(\Phi_{h}-\Phi\right)}}, & \text { case i } \\ \frac{\tilde{C} \alpha}{\tilde{\gamma}} \Phi^{-\alpha} \frac{1-e^{-\tilde{\gamma}\left(\Phi_{h}-\Phi\right)}\left(\Phi_{h} / \Phi\right)^{-\alpha-1}}{1-e^{-\tilde{\gamma}\left(\Phi_{h}-\Phi\right)}}, & \text { case ii }\end{cases}
$$


where we defined,

$$
\tilde{\gamma}=\frac{\gamma(d-1)}{\sqrt{d}}, \quad \tilde{C}=C \frac{d-1}{2 \sqrt{d}},
$$

and the coefficients $C, \kappa$ and $\alpha$ are defined in (3.1) and (3.2). The expression for $Y$ follows from the leading term in (3.10):

$$
Y_{0}(\Phi)=\frac{d-1}{d} \frac{1}{e^{2 \frac{(d-1) \gamma}{d}\left(\Phi_{h}-\Phi\right)}-1} .
$$

One can also obtain the differential equation for $\delta Y$ from the sub-leading term in (3.10) but we shall not need it here. The correction term in (3.14) follows from the expression for $\delta X$ above. One obtains,

$$
\delta A(\Phi)= \begin{cases}\frac{\tilde{C}}{\kappa+\tilde{\gamma}} e^{-\kappa \Phi}+\cdots, & \text { case } \mathrm{i} \\ -\frac{C}{2} \Phi^{-\alpha}+\cdots, & \text { case } \mathrm{ii}\end{cases}
$$

in the limit $\Phi_{h} \rightarrow \infty$.

\section{Expressing $T_{c}$ and $\Phi_{\infty}$ in terms of $V_{\infty}$}

Using equation (B.3), we express $A_{s}$ as,

$$
e^{2 A_{s}\left(\Phi_{h}\right)}=e^{2 A\left(\Phi_{h}\right)+\frac{4}{d-1} \Phi_{h}}=\frac{T^{2}}{V\left(\Phi_{h}\right)^{2}}\left(\frac{4 \pi(d-1)}{\ell}\right)^{2} e^{\frac{4}{d-1} \Phi_{h}-2 \gamma \int_{0}^{\Phi_{h}} X} .
$$

We consider the large $\Phi_{h}$ limit of this equation. The potential $V(\Phi)$ in this limit, is given by (2.22). On the other hand, we can represent the potential $V(\Phi)$ (for any $\Phi$ ) by equation (A.13). In the limit $\Phi_{h} \rightarrow \infty$ the function $X$ asymptotes to $-1 / \sqrt{d}$ as in (C.1). Thus, by comparing (A.13) and (2.22) in this limit one finds, ${ }^{36}$

$$
e^{-2 \gamma \int_{0}^{\Phi_{h}} X}=V_{\infty} \frac{\ell^{2}}{(d-1)^{2}} e^{\sqrt{\frac{\xi}{d-1}} \Phi_{h}} .
$$

In the NCST case the normalization of $\Phi$ is given by $\xi=4 /(d-1)$. Then, substitution of (D.2) in (D.1), use of (2.22) and the fact that $T \rightarrow T_{c}$ in this limit one finds,

$$
\lim _{\Phi_{h} \rightarrow \infty} e^{2 A_{s}\left(\Phi_{h}\right)}=\frac{\left(4 \pi T_{c}\right)^{2}}{V_{\infty}} .
$$

On the other hand we know that $A_{s}\left(\Phi_{h}\right) \rightarrow 0$ in this limit. Thus, one finds,

$$
T_{c}=\frac{\sqrt{V_{\infty}}}{4 \pi}
$$

or

$$
V_{\infty}=\left(4 \pi T_{c}\right)^{2}
$$

${ }^{36}$ Using also the fact that the functions $X(\Phi)$ and $X_{0}(\Phi)$ become the same in the limit $\Phi_{h} \rightarrow \infty$. 
To express $\Phi_{\infty}$ in terms of $V_{\infty}$ we use the e.o.m. for $\Phi,($ A.6). We first recall that the constant $\Phi_{\infty}$ is defined as,

$$
\lim _{r \rightarrow \infty} \Phi(r)=\Phi_{\infty} r
$$

Use of (A.8) in (A.6) gives,

$$
\frac{d \Phi}{d r}=-\frac{d}{\ell \gamma} e^{A(\Phi)} X(\Phi) e^{-\gamma \int_{0}^{\Phi} X} .
$$

Now, using (D.2), (C.1) and the fact that $\xi=4 /(d-1)$,in the NCST case, one finds in the limit $\Phi_{h} \rightarrow \infty$,

$$
\left.\lim _{\Phi_{h} \rightarrow \infty} \frac{d \Phi}{d r}\right|_{\Phi=\Phi_{h}}=\Phi_{\infty}=\frac{\sqrt{d V_{\infty}}}{\gamma(d-1)} .
$$

Using the definition (A.2) and $\xi=4 /(d-1)$, one finally arrives at,

$$
\Phi_{\infty}=\frac{\sqrt{V_{\infty}}}{2} .
$$

From this one can also express $A_{\infty}$ in terms of $V_{\infty}$. We recall the definition of $A_{\infty}$,

$$
\lim _{r \rightarrow \infty} A(r)=-A_{\infty} r .
$$

Comparison of (2.21) and (D.10) gives,

$$
A_{\infty}=\frac{2}{d-1} \Phi_{\infty}=\frac{\sqrt{V_{\infty}}}{d-1}=\frac{4 \pi}{d-1} T_{c}
$$

where the last equation follows from (D.5).

\section{E Curvature invariants}

Einstein's equations that follow from (2.1) read,

$$
R_{\mu \nu}-\frac{1}{2} g_{\mu \nu} R=\xi\left(\partial_{\mu} \Phi \partial^{\mu} \Phi-\frac{1}{2} g_{\mu \nu}(\partial \Phi)^{2}\right)+\frac{1}{2} g_{\mu \nu} V(\Phi) .
$$

Tracing both sides one obtains,

$$
R=\xi(\partial \Phi)^{2}-\frac{d+1}{d-1} V(\Phi) .
$$

One can express the right hand side in terms of the scalar variables of appendix A. Substituting (A.6) and (A.10) in (E.2) one finds,

$$
R=\frac{d}{\ell^{2}}\left[(d-1) X^{2}-(d+1)\left(1+Y-X^{2}\right) e^{\gamma \int_{0}^{\Phi} \frac{Y}{X}}\right] e^{-2 \gamma \int_{0}^{\Phi} X} .
$$

Both for the TG solution and for the BH solution in the limit $\Phi_{h} \gg 1$ (which corresponds to the phase transition region $T \approx T_{c}$ ) one has, $Y \approx 0, X \approx X_{0}$ for all $\Phi{ }^{37}$ This is indeed the limit that we are interested in. Equation (E.3) becomes,

$$
R \approx \frac{d}{\ell^{2}}\left[2 d X_{0}^{2}-(d+1)\right] e^{-2 \gamma \int_{0}^{\Phi} X_{0}} .
$$

\footnotetext{
${ }^{37}$ Except at strict $\Phi=\Phi_{h}$ where in the $\mathrm{BH}$ case $Y$ has a singularity at this point.
} 
The approximation becomes better and better as $\Phi_{h}$ gets closer to infinity in the BH case. In the TG case (E.4) is exact. We are interested in how $R$ scales with $\Phi$ for large $\Phi$. The biggest contribution come from the large $\Phi$ region where we can use the asymptotic expression for the function $X,\left(\right.$ C.1): $X_{0} \rightarrow-1 / \sqrt{d}$. Substituting this in (E.4) and using (D.2) $\left(X(\Phi)\right.$ and $X_{0}(\Phi)$ become the same functions in the limit $\left.\Phi_{h} \gg 1\right)$, one finally arrives at,

$$
R \approx-\frac{d V_{\infty}}{d-1} e^{\frac{2 \sqrt{\xi}}{\sqrt{d-1}} \Phi}, \quad \text { as } \Phi \gg 1
$$

To repeat, in the $\mathrm{BH}$ case the approximation is valid in the region $\Phi_{h} \gg 1$. We see that $R$ is proportional to $V$ in the IR region. The same is true for the dilaton invariant $(\partial \Phi)^{2}$. One can also show that the higher order invariants such as $R_{\mu \nu \alpha \beta} R^{\mu \nu \alpha \beta}$ also scale as (powers) of $V$ in the IR.

One can calculate the string frame invariants from the Einstein-frame ones by passing to the string frame metric,

$$
g_{s, \mu \nu}=g_{\mu \nu} e^{\frac{4}{d-1} \Phi}
$$

Ricci scalar transforms as,

$$
R_{s}=e^{-\frac{4}{d-1} \Phi}\left[R+4 \frac{d}{d-1} \partial_{\mu} \partial^{\mu} \Phi+4 \frac{d}{d-1} \partial_{\mu} \Phi \partial_{\mu} \Phi\right]
$$

where the indices are raised and lowered by the Einstein-frame metric on the r.h.s. . Let us specify to the case of NCST normalization $\xi=4 /(d-1)$ where also the relations $(2.28)$ hold. Using (E.5) and (2.28) we see that the first and the last terms in the square brackets cancel in the leading order, wheras the second term scales exactly as the subleading pieces in the first and the last terms. Therefore one finds,

$$
R_{s} \propto \partial_{r}^{2} \delta \Phi(r)
$$

where $\delta \Phi$ is the subleading piece in the IR: $\Phi \rightarrow \Phi_{\infty} r+\delta \Phi(r)$. In order to find this, one works out equation (D.7) to sub-leading order by substituting $X$ in (C.1) and using (C.2) in the limit $\Phi_{h} \rightarrow \infty$. One then finds,

$$
\delta \Phi \propto e^{-\kappa \Phi} \Phi_{\infty}
$$

in case $\mathrm{i}$ and,

$$
\delta \Phi \propto r^{-\alpha}
$$

in case ii. Substituion in (E.8) finally yields,

$$
\left.R_{s} \propto e^{-\kappa \Phi}, \quad(\text { case } i) ; \quad \Phi^{-\alpha}, \quad \text { (case } i i\right),
$$

in the limit $\Phi \rightarrow \infty$. We see that the string frame Ricci scalar vanishes near the transition region. The same is true for the dilaton invariant $(\partial \Phi)^{2}$ in the string frame. Higher order invariants vanish with a stronger rate. This shows that the $\alpha^{\prime}$ corrections in the string frame become unimportant near the transition region. 


\section{F Bulk fluctuations and dissipation}

In this section, we present the details of the calculations in section 5.2. We first derive the fluctuation equation (5.8) that we reproduce here:

$$
h_{b}^{\prime \prime}+h_{b}^{\prime}\left(\frac{2 \xi}{d-1} \frac{1}{A^{\prime}}+(d-2) A^{\prime}-3 B^{\prime}+\frac{f^{\prime}}{f}\right)+h_{b}\left(\frac{\omega^{2}}{f^{2}} e^{2 B-2 A}+\frac{f^{\prime}}{f} B^{\prime}-\frac{\xi}{d-1} \frac{f^{\prime}}{f A^{\prime}}\right)=0,
$$

where prime denotes the derivative w.r.t. $\Phi$ and the metric functions are defined in the $\Phi$-frame:

$$
d s^{2}=e^{2 A(\Phi)}\left(-f(\Phi) d t^{2}+d x_{d-1}^{2}\right)+e^{2 B(\Phi)} \frac{d \Phi^{2}}{f(\Phi)} .
$$

One fluctuates the Einstein's e.o.m. with the metric fluctuations:

$$
\begin{aligned}
g_{00} & =-e^{2 A} f\left(1+\frac{\epsilon}{2} h_{00} e^{-i \omega t}\right)^{2}, \\
g_{i i} & =e^{2 A}\left(1+\frac{\epsilon}{2} h_{b} e^{-i \omega t}\right)^{2}, \\
g_{\Phi \Phi} & =\frac{e^{2 B}}{f}\left(1+\frac{\epsilon}{2} h_{\Phi \Phi} e^{-i \omega t}\right)^{2},
\end{aligned}
$$

and $\delta \Phi=0$. The last choice corresponds to the gauge (F.2). We need only two of the zeroth order Einstein eqs. in this gauge:

$$
\begin{aligned}
f^{\prime \prime}+d A^{\prime} f^{\prime}-B^{\prime} f^{\prime} & =0 \\
A^{\prime \prime}-A^{\prime} B^{\prime}+\frac{\xi}{d-1} & =0 .
\end{aligned}
$$

The fluctuation equations for $h_{00}, h_{b}$ and $h_{\Phi \Phi}$ are obtained by expanding the Einstein eqs. $G_{\mu}^{\nu}=T_{\mu}^{\nu}$ to order $\epsilon$ where the stress tensor at this order reads:

$$
\left.T_{\mu}^{\nu}\right|_{\epsilon}=-\xi e^{-2 B} f h_{\Phi \Phi} e^{-i \omega t}\left(\delta_{\Phi}^{\nu} \delta_{\mu}^{\Phi}-\frac{1}{2} \delta_{\mu}^{\nu}\right) .
$$

One also needs to expand the Einstein tensors to this order. This is done with help of Mathematica. We shall present the final results. From the first order terms in $\epsilon$ in the Einstein's equations one finds the following e.o.m for the fluctuations: From the $\{\Phi, \Phi\}$ Einstein equation one finds,

$$
h_{00}^{\prime}=h_{\Phi \Phi}\left(-\frac{\xi}{d-1} \frac{1}{A^{\prime}}+d A^{\prime}+\frac{f}{f^{\prime}}\right)-h_{b}^{\prime}\left(d-1+\frac{f^{\prime}}{2 A^{\prime} f}\right)-\frac{\omega^{2}}{f^{2}} \frac{e^{2 B-2 A}}{A^{\prime}} h_{b} .
$$

From the $\{0, \Phi\}$ Einstein equation one finds,

$$
h_{\Phi \Phi}=\frac{1}{A^{\prime}}\left(h_{b}^{\prime}-\frac{f^{\prime}}{2 f} h_{b}\right) .
$$

Finally, from the $\{i, i\}$ Einstein equation one finds a complicated expression that involves $h_{b}, h_{b}^{\prime}$ and $h_{b}^{\prime \prime}$ that simplifies upon use of (F.7), (F.8) and the zeroth order eqs. (F.4) and (F.5) and reduces to (F.1). 
Now, we move on to the details that lead to (5.7) that we reproduce here:

$$
\operatorname{Im} J=\frac{i}{4} \xi \frac{f}{A^{\prime 2}} e^{d A-B}\left(h_{b}^{*} h_{b}^{\prime}-h_{b} h_{b}^{*^{\prime}}\right) .
$$

This is obtained by expanding the Lagrangian (2.1) to second order in $\epsilon$ in fluctuations, as explained in [22], as follows:

Define the fluctuation vectors $\vec{h}=\left(h_{00}, h_{b}, h_{\Phi \Phi}\right)$. Then the $\mathcal{O}\left(\epsilon^{2}\right)$ term in the Lagrangian is of the form,

$$
\mathcal{L}=\frac{1}{2} \dot{h}^{T} M_{t t} h+\frac{1}{2} h^{\prime T} M_{\Phi \Phi} h^{\prime}+h^{\prime T} M_{\Phi} h+\frac{1}{2} h^{T} M h+\cdots
$$

where the ellipsis refer to total derivative terms in $\Phi$ and in t. Now, one formally promotes $\vec{h}$ to complex valued fields and writes ( $h$ should be understood as a complex vector in the following):

$$
\mathcal{L}=h^{\dagger^{\prime}} M_{\Phi \Phi} h^{\prime}+h^{\dagger^{\prime}} M_{\Phi} h+\cdots
$$

where remaining terms are unimportant as they do not contribute to $I m J$ which is the conserved flux under the $\mathrm{U}(1)$ rotations $\vec{h} \rightarrow e^{i \theta} \vec{h}$ :

$$
\mathcal{L}=\partial_{\Phi} J+\cdots, \quad J=h^{\dagger}\left(M_{\Phi \Phi} h^{\prime}+\left(M_{\Phi}+\cdots\right) h\right) .
$$

Here the ellipsis in $\mathcal{L}$ refer to terms that vanish on shell and the ellipsis in $J$ refer to a symmetric matrix that vanish in the imaginary part of $J$ hence irrelevant for our purpose.

Thus the entire calculation is reduced to the computation of the $3 \times 3$ matrices $M_{\Phi \Phi}$ and $M_{\Phi}$. Clearly, only the $\sqrt{g} R$ piece of (2.1) will give rise to terms with single or double derivatives in $\Phi$, as in (F.10). Moreover, we are not interested in the t-dependence, therefore one can ignore the time dependence in the fluctuations (F.3). One expands $\sqrt{g} R$ with help of a symbolic computer code and obtains $M_{\Phi \Phi}$ and $M_{\Phi}$ as (after integration by parts in the double derivative terms and upon use of the zeroth order eqs. (F.4) and (F.5)):

$$
\begin{aligned}
M_{\Phi \Phi} & =(d-1) f e^{d A-B}\left(\begin{array}{ccc}
0 & \frac{1}{2} & 0 \\
\frac{1}{2} & \frac{d-2}{2} & 0 \\
0 & 0 & 0
\end{array}\right) \\
M_{\Phi} & =\frac{(d-1)}{4} e^{d A-B}\left(\begin{array}{ccc}
* & -f^{\prime}-2 A^{\prime} f & \frac{f^{\prime}+2 A^{\prime} f}{d-1} \\
-2 f A^{\prime} & * & 2 f A^{\prime} \\
\frac{f^{\prime}+2 d A^{\prime} f}{d-1} & f^{\prime}+2 d A^{\prime} f & *
\end{array}\right)
\end{aligned}
$$

where the order of the components are according to $\vec{h}=\left(h_{00}, h_{b}, h_{\Phi \Phi}\right)$ and the stars refer to terms that do not contribute to the imaginary part of the flux. One calculates the flux from (F.12). It reduces to (F.9) upon use of the first order equations (F.7) and (F.8).

Open Access. This article is distributed under the terms of the Creative Commons Attribution Noncommercial License which permits any noncommercial use, distribution, and reproduction in any medium, provided the original author(s) and source are credited. 


\section{References}

[1] S.W. Hawking and D.N. Page, Thermodynamics of black holes in Anti-de Sitter space, Commun. Math. Phys. 87 (1983) 577 [SPIRES].

[2] E. Witten, Anti-de Sitter space, thermal phase transition and confinement in gauge theories, Adv. Theor. Math. Phys. 2 (1998) 505 [hep-th/9803131] [SPIRES].

[3] J.M. Maldacena, The large $N$ limit of superconformal field theories and supergravity, Adv. Theor. Math. Phys. 2 (1998) 231 [Int. J. Theor. Phys. 38 (1999) 1113] [hep-th/9711200] [SPIRES].

[4] S.S. Gubser, I.R. Klebanov and A.M. Polyakov, Gauge theory correlators from non-critical string theory, Phys. Lett. B 428 (1998) 105 [hep-th/9802109] [SPIRES].

[5] E. Witten, Anti-de Sitter space and holography, Adv. Theor. Math. Phys. 2 (1998) 253 [hep-th/9802150] [SPIRES].

[6] U. Gürsoy, E. Kiritsis, L. Mazzanti and F. Nitti, Deconfinement and gluon plasma dynamics in improved holographic QCD, Phys. Rev. Lett. 101 (2008) 181601 [arXiv:0804.0899] [SPIRES].

[7] U. Gürsoy and E. Kiritsis, Exploring improved holographic theories for QCD: part I, JHEP 02 (2008) 032 [arXiv:0707.1324] [SPIRES].

[8] U. Gürsoy, E. Kiritsis and F. Nitti, Exploring improved holographic theories for QCD: part II, JHEP 02 (2008) 019 [arXiv:0707.1349] [SPIRES].

[9] U. Gürsoy, E. Kiritsis, L. Mazzanti and F. Nitti, Holography and thermodynamics of $5 D$ dilaton-gravity, JHEP 05 (2009) 033 [arXiv:0812.0792] [SPIRES].

[10] A. Buchel, Critical phenomena in $N=4$ SYM plasma, Nucl. Phys. B 841 (2010) 59 [arXiv:1005.0819] [SPIRES].

[11] U. Gursoy, to appear.

[12] J.M. Kosterlitz and D.J. Thouless, Ordering, metastability and phase transitions in two-dimensional systems, J. Phys. C 6 (1973) 1181 [SPIRES].

[13] V.L. Berezinsky, Destruction of long range order in one-dimensional and two-dimensional systems having a continuous symmetry group. 1. Classical systems, Sov. Phys. JETP 32 (1971) 493 [SPIRES].

[14] K. Jensen, A. Karch, D.T. Son and E.G. Thompson, Holographic Berezinskii-Kosterlitz-Thouless transitions, Phys. Rev. Lett. 105 (2010) 041601 [arXiv: 1002.3159] [SPIRES].

[15] N. Iqbal, H. Liu, M. Mezei and Q. Si, Quantum phase transitions in holographic models of magnetism and superconductors, Phys. Rev. D 82 (2010) 045002 [arXiv: 1003.0010] [SPIRES].

[16] G. Clement, J.C. Fabris and G.T. Marques, Hawking radiation of linear dilaton black holes, Phys. Lett. B 651 (2007) 54 [arXiv:0704.0399] [SPIRES].

[17] J. Polchinski, String theory. Volume I, Cambridge University Press, Cambridge U.K. (2005).

[18] B.I. Halperin and D.R. Nelson, Theory of two-dimensional melting, Phys. Rev. Lett. 41 (1978) 121.

[19] W. Janke, D.A. Johnston and R. Kenna, Properties of higher-order phase transitions, Nucl. Phys. B 736 (2006) 319 [SPIRES]. 
[20] G. Policastro, D.T. Son and A.O. Starinets, The shear viscosity of strongly coupled $N=4$ supersymmetric Yang-Mills plasma, Phys. Rev. Lett. 87 (2001) 081601 [hep-th/0104066] [SPIRES].

[21] A. Buchel and J.T. Liu, Universality of the shear viscosity in supergravity, Phys. Rev. Lett. 93 (2004) 090602 [hep-th/0311175] [SPIRES].

[22] S.S. Gubser, S.S. Pufu and F.D. Rocha, Bulk viscosity of strongly coupled plasmas with holographic duals, JHEP 08 (2008) 085 [arXiv:0806.0407] [SPIRES].

[23] U. Gürsoy, E. Kiritsis, G. Michalogiorgakis and F. Nitti, Thermal transport and drag force in improved holographic QCD, JHEP 12 (2009) 056 [arXiv:0906.1890] [SPIRES].

[24] R. Kubo, Statistical-mechanical theory of irreversible processes. I. General theory and simple applications to magnetic and conduction problems, J. Phys. Soc. Japan 12 (1957) 570.

[25] J.M. Kosterlitz, The critical properties of the two-dimensional xy model, J. Phys. C 7 (1974) 1046 [SPIRES].

[26] E. D'Hoker and D.Z. Freedman, Supersymmetric gauge theories and the AdS/CFT correspondence, hep-th/0201253 [SPIRES].

[27] H.A. Chamblin and H.S. Reall, Dynamic dilatonic domain walls, Nucl. Phys. B 562 (1999) 133 [hep-th/9903225] [SPIRES].

[28] R.M. Wald, Black hole entropy is the Noether charge, Phys. Rev. D 48 (1993) 3427 [gr-qc/9307038] [SPIRES].

[29] S. Bhattacharyya, S. Minwalla and K. Papadodimas, Small hairy black holes in $A d S_{5} \times S^{5}$, arXiv: 1005.1287 [SPIRES].

[30] U. Gürsoy, E. Kiritsis, L. Mazzanti and F. Nitti, Improved holographic Yang-Mills at finite temperature: comparison with data, Nucl. Phys. B 820 (2009) 148 [arXiv:0903.2859] [SPIRES]. 